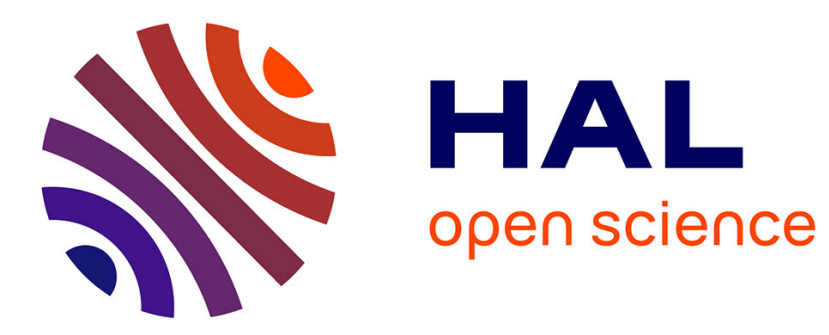

\title{
Two-scale thermomechanical damage model for dynamic shear failure in brittle solids
}

Kokouvi Gbetchi, Cristian Dascalu

\section{To cite this version:}

Kokouvi Gbetchi, Cristian Dascalu. Two-scale thermomechanical damage model for dynamic shear failure in brittle solids. Continuum Mechanics and Thermodynamics, 2021, 33, pp.445-473. 10.1007/s00161-020-00916-x . hal-02915361

\section{HAL Id: hal-02915361 \\ https://hal.univ-lorraine.fr/hal-02915361}

Submitted on 14 Aug 2020

HAL is a multi-disciplinary open access archive for the deposit and dissemination of scientific research documents, whether they are published or not. The documents may come from teaching and research institutions in France or abroad, or from public or private research centers.
L'archive ouverte pluridisciplinaire HAL, est destinée au dépôt et à la diffusion de documents scientifiques de niveau recherche, publiés ou non, émanant des établissements d'enseignement et de recherche français ou étrangers, des laboratoires publics ou privés. 


\title{
Two-scale thermomechanical damage model for dynamic shear failure in brittle solids
}

\author{
Kokouvi Gbetchi · Cristian Dascalu
}

Received : date / Accepted : date

Résumé A coupled thermo-mechanical damage approach for dynamic shear failure in brittle solids is proposed in the present contribution. The model is constructed by asymptotic homogenization from microstructures with dynamically evolving microcracks, in mode II, with unilateral contact and friction conditions on their lips. Crack-tip and frictional heating effects assumed at the small-scale give rise to distributed heat sources in the macroscopic temperature equation and specific dissipation terms in the upscaled damage law. The analysis of the effective thermomechanical response of the model reveals strain-rate and size effects and the influence of friction and growth of microcracks on the macroscopic thermal evolutions.

Keywords Microcracks · Mode II dynamic propagation · Frictional and crack-tip heating · Homogenization · Damage law · Macroscopic response simulations

\section{Introduction}

The material response of quasi-brittle solids under compression loadings involving complex features like hardening and softening, stiffness degradation, induced anisotropy, irreversible deformations and thermal changes are the result of microscopic evolutions of microcracks with frictional sliding on their lips. The dynamic behavior of these materials is often associated with important thermal changes originated by small-scale dissipation processes related to microcrack propagation and frictional heating. In order to properly account for such mechanisms and their coupling, a multiscale modeling framework is well suited. The objective of the present contribution is to construct a two-scale thermomechanical damage model based on dynamic evolutions of mode II microcracks with associated heat dissipation effects due to their propagation and frictional sliding.

Micromechanical approaches for stationary or evolving frictional micro-cracks have been developed, among others, in [29], [34], [2], [4], [27], [35], [51], [33], [24],

K. Gbetchi · C. Dascalu

Université de Lorraine, CNRS, Arts et Métiers ParisTech, LEM3, F-57000 Metz, France

E-mail: cristian.dascalu@univ-lorraine.fr 
[26], [32], [54], [55]. In dynamics, models based on micro-mechanics have been developed to study the compressive failure response of brittle solids in the case when the main microscopic mechanism is the tensile fracture mode. A study of an array of interacting and dynamically growing wing cracks and the corresponding ratedependent dynamic damage evolution has been performed in [36]. In [40] the authors developed a micro-mechanical model for ceramics based on non-interacting, uniformly distributed sliding micro-cracks subjected to dynamic compressive loading and predicted effects of the rate sensitivity on failure strength. A model that combines damage evolution theory with dynamic crack growth under uniaxial dynamic compressionhas been proposed in [28]. Also, in [37] a model based on the evolution of tensile wing micro-cracks in the case of uniaxial compression under constant strain loading is developed. A more recent dynamic damage model inspired by the same wing crack mechanism and valid over a wide range of loading rates has been proposed in [8]. In [47] a phase-field fracture model has been used to simulate the intersonic shear fracture of brittle specimens under impact [45].

Thermal effects play an important role in the description of dynamic failure. Temperature measurements for opening or shear mode cracks, like those reported in $[42,43,10,12]$ for brittle polymers, revealed a significant temperature rise in a highly localized area surrounding the crack tip during propagation. Attempts to model such heating effects in the context of Fracture Mechanics have been made for instance in $[7,49]$ by considering crack models with Dirac-type heat sources located at the tip point and which intensity represents the excess of energy release rate with respect to that necessary for the new crack surface formation. In [10] the authors proposed a thermally dissipative cohesive zone model for dynamic fracture of amorphous polymers. Their model is based on the assumption that the excess supplied energy in the crack-tip region is converted to heat within the cohesive zone that models the crazing process. More recently, a multiscale damage approach has obtained [16] for mode I dynamic failure with moving heat sources at the tips of microcracks.

A second source of heating for shear rupture is the frictional sliding on the crack lips. For brittle materials under impact loading, the frictional heating on cracks has been considered for instance in [13]. Earthquakes motion is generally associated with the development of important densities of microcracks and the frictional heating effects play an important role in the description of seismic events $[31,20,41]$. In [17] a statistical approach has been developed to predict the dynamic response of brittle reactive materials with myriad of microcracks under impact and including the hot spot formation by frictional heating. A phase field damage model that includes heat sources due to frictional heating at the crack surfaces and heat dissipation during crack propagation has been used in $[25,18]$ to illustrate the nucleation of hot spots in energetic materials.

Impact experiments reported in $[44,45,39,9,10]$ on bonded or side-grooved sam-ples of brittle polymers revealed that under high strain rate loadings a failure mode transition from opening to shear fracture takes place. In contrast with the mode I crack velocities that are much smaller than the theoretical limit, the Rayleigh wave speed $c_{R}$, the shear cracks may evolve rapidly up to $c_{R}$ and may pass in the supershear regime, with tip velocities $v$ larger than the shear wave speed $c_{S}$. The microstructural observations of the fracture surfaces [39,3], for mode II failure of PMMA samples under impact, revealed the existence of complex morphologies which do not correspond to en echelon mode I microcracks, but rather 
to a mode II process zone. It was also observed [9,12] that frictional contact plays an important role during the shear failure, leading to temperature rise up to the glass transition temperature and polymer melting effects. Similarly to the mode I case [10,12], substantial heating due to fracture growth has been measured at the tip of a mode II crack $[9,12]$. These experimental results show the necessity of appropriate modeling of the thermal dissipation mechanisms related to dynamic crack propagation and friction in mode II failure and this represents the main motivation of the present contribution. We investigate here the possibility of modeling the shear failure of brittle solids using a thermomechanical damage model obtained by upscaling of microstructures with rapidly evolving mode II microcracks, including frictional and crack growth thermal dissipation effects.

A method to obtain damage evolution laws from microcracks propagation criteria, using the asymptotic homogenization technique, has been proposed in [15]. In dynamics, a damage model has been established for mode I microcracks in [30, 14] and heat dissipation effects have been included in the mode I dynamic damage approach in [16]. For microcracks with contact and friction, a quasi-static damage law has been obtained in [53] and it has been extended to dynamics in [5]. The purpose of the present work is the construction of a two-scale thermomechanical damage model by homogenization in the case of dynamic mode II evolutions of microcracks having contact and friction conditions on their lips and with crack-tip and frictional heating sources.

The paper is organized as follows. In section 2 , the thermoelastic dynamic fracture problem is formulated. The next two sections are devoted to the homogenization analysis for the macroscopic temperature and elastodynamics equations for the microcracked solid and the dynamic damage law deduced from the microscopic propagation criterion. The analysis of the local macroscopic response of the new model is performed in Section 5 for the effective coefficients, the influence of small-scale friction, the strain-rate and microstructural size, the thermal evolutions accompanying the damage processes. Results of numerical simulations for dynamic shear rupture of PMMA samples under impact loading and the associated thermal evolutions are presented and compared with the experimental data in Section 6 .

\section{Thermoelastic fracture problem formulation}

For a solid containing a large number of cracks that are small with respect to the size of the whole structure, we distinguish between the macroscopic properties at the scale of the solid body and the microscopic ones characterizing the local behavior in the vicinity of one microcrack.

The distribution of microcracks in the solid $B$ is assumed to be locally periodic, as illustrated in Figure 1, all of them being straight and parallel to the $x_{1}$ axis. We denote by $l$ the microcrack length and by $l_{c}$ the size of the period. The local periodicity means slow spatial variations of $l$, with fixed $l_{c}$, such that locally the microstructure may be considered periodic.

If $L_{c}$ is a characteristic length of the solid body $B$, as in Fig. 1, then the small parameter $\varepsilon=\frac{l_{c}}{L_{c}}$, is a mesure of the difference between microscopic and macroscopic scales. The mechanical and thermal fields describing the corresponding two-scale multi-fracture problem will be denoted with an upper index $\varepsilon$. 
a)

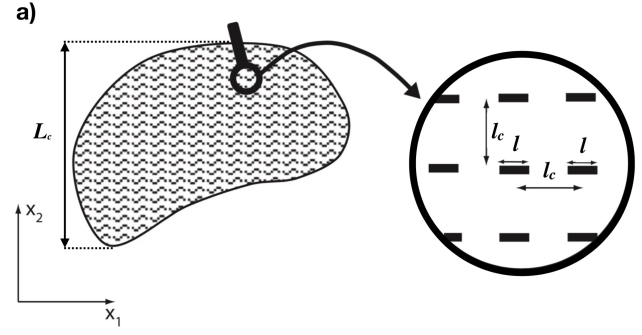

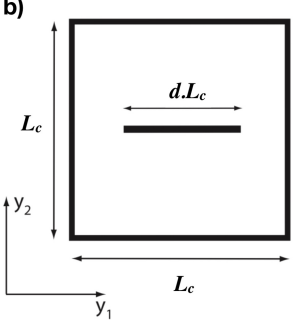

FiguRE 1: a) Solid body with locally periodic microstructure with $l_{c}$ the size of a period, $l$ the micro-crack length and $L_{c}$ the characteristic length of the macrostructure. b) Reference cell of size $L_{c}$ with rescaled crack length $d . L_{c}$.

The thermo-mechanical evolutions in a material point of the solid are described by the nonlinear equations of dynamic thermoelasticity

$$
\begin{aligned}
c \frac{\partial T^{\varepsilon}}{\partial t}+b_{i j} T^{\varepsilon} \frac{\partial e_{x i j}\left(\mathbf{u}^{\varepsilon}\right)}{\partial t}+\frac{\partial q_{j}^{\varepsilon}}{\partial x_{j}} & =0 \\
\rho \frac{\partial^{2} u_{i}^{\varepsilon}}{\partial t^{2}}-\frac{\partial \sigma_{i j}^{\varepsilon}}{\partial x_{j}} & =0
\end{aligned}
$$

where $c$ is the specific heat and $\rho$ the density of the material, $b_{i j}=\alpha(3 \lambda+2 \mu) \delta_{i j}$ is the tensor of isotropic thermoelastic coupling coefficients, with $\lambda, \mu$ the Lamé constants and $\alpha$ the thermal expansion coefficient. The strain tensor calculated with respect to $x_{i}$ variables is denoted by $e_{x i j}\left(\mathbf{u}^{\varepsilon}\right)=\frac{1}{2}\left(\frac{\partial u_{i}^{\varepsilon}}{\partial x_{j}}+\frac{\partial u_{j}^{\varepsilon}}{\partial x_{i}}\right)$.

The heat flux vector $\mathbf{q}^{\varepsilon}$ is given by the Fourier law :

$$
q_{j}^{\varepsilon}=-k \frac{\partial T^{\varepsilon}}{\partial x_{j}}
$$

where $k$ is the thermal conduction coefficient of the material, while the thermoelastic constitutive relations for stresses are

$$
\sigma_{i j}^{\varepsilon}=a_{i j k l} e_{x k l}\left(\mathbf{u}^{\varepsilon}\right)-b_{i j}\left(T^{\varepsilon}-T_{0}\right)
$$

with $a_{i j k l}=\lambda \delta_{i j} \delta_{k l}+\mu\left(\delta_{i k} \delta_{j l}+\delta_{i l} \delta_{j k}\right)$ being the isotropic elastic moduli.

On each crack faces unilateral contact conditions are assumed :

$$
\left[\boldsymbol{\sigma}^{\varepsilon} \mathbf{N}\right]=0 ; \quad \mathbf{N} \boldsymbol{\sigma}^{\varepsilon} \mathbf{N}<0 ; \quad\left[\mathbf{u}^{\varepsilon} \mathbf{N}\right]=0
$$

together with the Coulomb friction law :

$$
\left|\mathbf{T} \boldsymbol{\sigma}^{\varepsilon} \mathbf{N}\right|=-\mu_{f} \mathbf{N} \boldsymbol{\sigma}^{\varepsilon} \mathbf{N}
$$

In these relations, $\mathbf{N}$ is the unit vector normal to the crack, as shown in Fig. 2, $\mathbf{T}$ is a unit vector tangent to the crack line, $\mu_{f}$ is the friction coefficient and [.] indicates the jump across the crack faces.

The frictional sliding between the crack faces gives rise to heating. A superficial heat source $[56,57]$ equal to the frictional power, calculated with the shear stress 
and the relative sliding velocity, is considered together with the continuity of the temperature field across the crack surface :

$$
\left[\boldsymbol{q}^{\varepsilon} \mathbf{N}\right]=\mu_{f} \mathbf{N} \boldsymbol{\sigma}^{\varepsilon} \mathbf{N}\left[\frac{\partial \mathbf{u}^{\varepsilon}}{\partial t} \mathbf{T}\right] ; \quad\left[T^{\varepsilon}\right]=0
$$

The sign in the right member of the first relation corresponds to negative relative velocities, as will be the case in the examples of the last section.

A second dissipation effect is linked to crack propagation. Most of the energy dissipated for rupture is converted into heat and the crack tips are behaving like moving heat sources $[7,49,16]$. If we define the heat flow from the crack tip as the limit

$$
D^{\varepsilon}=\lim _{r \rightarrow 0} \int_{\Gamma_{r}} \boldsymbol{q}^{\varepsilon} \mathbf{n} d s
$$

where $\Gamma_{r}$ is a small contour shrinking to the tip as represented in Fig. 2, then the local balance of energy during propagation reads

$$
D^{\varepsilon}=v_{p} \cdot \mathcal{G}^{d \varepsilon}
$$

with $v_{p}=\frac{1}{2} \frac{d l}{d t}$ being the crack-tip velocity. The term $\mathcal{G}^{d \varepsilon}$ represents the dynamic energy release-rate

$$
\mathcal{G}^{d \varepsilon}=\lim _{r \rightarrow 0} \int_{\Gamma_{r}}\left(\left(\mathcal{U}^{\varepsilon}+\mathcal{K}^{\varepsilon}\right) n_{1}-\sigma_{i j}^{\varepsilon} n_{j} \frac{\partial u_{i}^{\varepsilon}}{\partial x_{1}}\right) d s
$$

calculated with the internal energy :

$$
\mathcal{U}^{\varepsilon}=\frac{1}{2} a_{i j k l} e_{x k l}\left(\mathbf{u}^{\varepsilon}\right) e_{x i j}\left(\mathbf{u}^{\varepsilon}\right)+b_{i j} T_{0} e_{x i j}\left(\mathbf{u}^{\varepsilon}\right)+c T^{\varepsilon}
$$

and the kinetic energy

$$
\mathcal{K}^{\varepsilon}=\frac{1}{2} \rho \frac{\partial \mathbf{u}^{\varepsilon}}{\partial t} \frac{\partial \mathbf{u}^{\varepsilon}}{\partial t}
$$

For the propagation of the cracks, we consider a Griffith-type criterion $\mathcal{G}^{d \varepsilon}=$ $\mathcal{G}_{c}$, with $\mathcal{G}_{c}$ the critical fracture energy. In this case the relation (9) becomes $D^{\varepsilon}=v_{p} \cdot \mathcal{G}_{c}$.

\section{Asymptotic homogenization analysis}

The previous multi-fracture problem will be upscaled to obtain an equivalent macroscopic formulation in this section. The asymptotic homogenization method $[46,21]$ will be used to deduce the macroscopic thermoelasticity equations from the initial problem described previously. A similar approach has been used for coupled dynamic thermoelasticity by $[19,22,38,6]$ in the case of solids without cracks. For thermoelastic microcracked solids in dynamics, the method has been used in [16] in the case of opening microcracks. In this section, we focus on the influence of the contact and frictional conditions on shearing microcracks and of the non-classical heat sources associated with friction and rupture dissipations. The analysis performed here for the coupled thermoelastic system will be completed in Section 4 with the construction of the thermoelastic damage law by homogenization. 


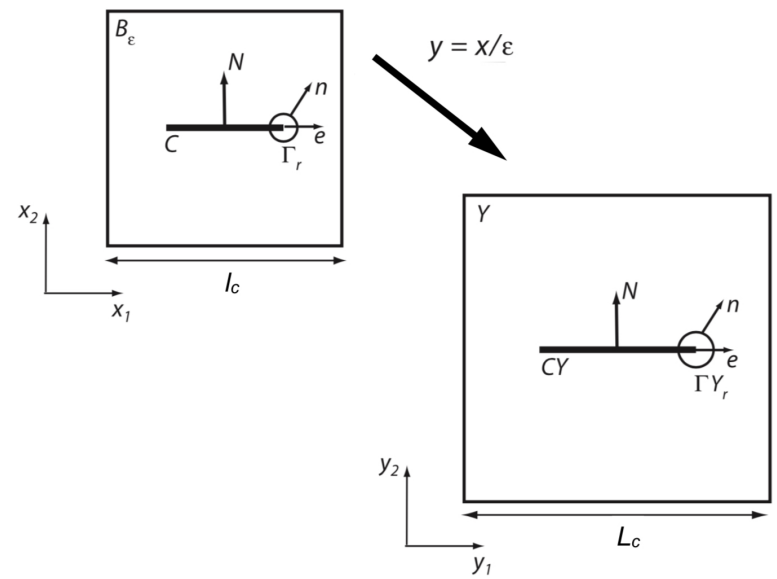

FIgURE 2: Scaling of the microscopic period $B_{\varepsilon}$ with a crack $C$ into the reference cell $Y$ with the crack $C Y$. The unit vectors $\mathbf{n}$ and e indicate the normal to the crack-tip contours $\Gamma_{r}$ and $\Gamma Y_{r}$ and, respectively, the propagation direction.

We introduce the damage variable $d=\frac{l}{l_{c}}$ as the microcrack length normalized by the size of the microscopic period. The local periodicity assumption for the length $l$, with constant $l_{c}$, will assure large-scale spatial variations of the damage variable $d$.

In what follows, we assume that the scale of microcracks is small in comparison with that of the solid body. This is expressed by the separation of scales condition

$$
\varepsilon=\frac{l_{c}}{L_{c}} \ll 1
$$

Small-scale variations of the thermomechanical fields do not have much influence at the large scale. Two different systems of space variables will be considered for the two scales and both should be taken into account in the description of the physical fields, being treated as independent variables.

We will distinguish between the macroscopic variable $\mathbf{x}$ and the microscopic variable $\mathbf{y}$, linked by the relation $\mathbf{y}=\frac{\mathbf{x}}{\varepsilon}$. For a field $f$ depending on both $\mathbf{x}$ and $\mathbf{y}$, the total spatial derivative can be calculated by the formula $\frac{d f}{d x_{i}}=\frac{\partial f}{\partial x_{i}}+\frac{1}{\varepsilon} \frac{\partial f}{\partial y_{i}}$. The scaling of a microscopic period $B_{\varepsilon}$, containing a crack $C$, by this transformation leads to a reference cell $Y$, of size $L_{c}$ and containing the crack $C Y$ of length $d . L_{c}$, as illustrated in Figure 2.

Asymptotic developments with respect to $\varepsilon$ for the temperature and displacement fields are considered :

$$
\begin{aligned}
\mathbf{u}^{\varepsilon}(\mathbf{x}, t) & =\mathbf{u}^{(0)}(\mathbf{x}, \mathbf{y}, t)+\varepsilon \mathbf{u}^{(1)}(\mathbf{x}, \mathbf{y}, t)+\varepsilon^{2} \mathbf{u}^{(2)}(\mathbf{x}, \mathbf{y}, t)+\ldots \\
T^{\varepsilon}(\mathbf{x}, t) & =T^{(0)}(\mathbf{x}, \mathbf{y}, t)+\varepsilon T^{(1)}(\mathbf{x}, \mathbf{y}, t)+\varepsilon^{2} T^{(2)}(\mathbf{x}, \mathbf{y}, t)+\ldots
\end{aligned}
$$

where $\mathbf{u}^{(i)}(x, y, t)$ and $T^{(i)}(x, y, t), \mathbf{x} \in B$ and $\mathbf{y} \in Y$ are $Y$-periodic. 
Using these expansions in the equations (3-4) we get for the heat flux and stress fields :

$$
\begin{array}{r}
\boldsymbol{q}^{\varepsilon}(\mathbf{x}, t)=\frac{1}{\varepsilon} \boldsymbol{q}^{(-1)}(\mathbf{x}, \mathbf{y}, t)+\boldsymbol{q}^{(0)}(\mathbf{x}, \mathbf{y}, t)+\varepsilon \boldsymbol{q}^{(1)}(\mathbf{x}, \mathbf{y}, t)+\varepsilon^{2} \boldsymbol{q}^{(2)}(\mathbf{x}, \mathbf{y}, t)+\ldots \\
\boldsymbol{\sigma}^{\varepsilon}(\mathbf{x}, t)=\frac{1}{\varepsilon} \boldsymbol{\sigma}^{(-1)}(\mathbf{x}, \mathbf{y}, t)+\boldsymbol{\sigma}^{(0)}(\mathbf{x}, \mathbf{y}, t)+\varepsilon \boldsymbol{\sigma}^{(1)}(\mathbf{x}, \mathbf{y}, t)+\varepsilon^{2} \boldsymbol{\sigma}^{(2)}(\mathbf{x}, \mathbf{y}, t)+\ldots
\end{array}
$$

The expressions of $\boldsymbol{q}^{(p)}(\mathbf{x}, \mathbf{y}, t)$ and $\boldsymbol{\sigma}^{(p)}(\mathbf{x}, \mathbf{y}, t)$ and are given in the Appendix A.

When these developments are replaced in the momentum and temperature equations (1-2) one obtains problems of order $\varepsilon^{n}$, with $n=-2,-1,0$, in the form :

$$
\begin{gathered}
\frac{\partial q_{j}^{(-1)}}{\partial y_{j}}=0 ; \frac{\partial \sigma_{i j}^{(-1)}}{\partial y_{j}}=0 \\
b_{i j} T^{(0)} \frac{\partial e_{y i j}\left(u^{(0)}\right)}{\partial t}+\frac{\partial q_{j}^{(-1)}}{\partial x_{j}}+\frac{\partial q_{j}^{(0)}}{\partial y_{j}}=0 ; \frac{\partial \sigma_{i j}^{(-1)}}{\partial x_{j}}+\frac{\partial \sigma_{i j}^{(0)}}{\partial y_{j}}=0 \\
c \frac{\partial T^{(0)}}{\partial t}+b_{i j} T^{(0)}\left(\frac{\partial e_{x i j}\left(u^{(0)}\right)}{\partial t}+\frac{\partial e_{y i j}\left(u^{(1)}\right)}{\partial t}\right)+\frac{\partial q_{j}^{(0)}}{\partial x_{j}}+\frac{\partial q_{j}^{(1)}}{\partial y_{j}}=0 \\
\rho \frac{\partial^{2} u^{(0)}}{\partial t^{2}}-\frac{\partial \sigma_{i j}^{(0)}}{\partial x_{j}}-\frac{\partial \sigma_{i j}^{(1)}}{\partial y_{j}}=0
\end{gathered}
$$

From the boundary conditions (5-6), for $m=-1,0,1$, we deduce :

$$
\begin{gathered}
{\left[\sigma_{i j}^{(m)} N_{j}\right]=0 ; \quad N_{i} \sigma_{i j}^{(m)} N_{j}<0 ; \quad\left[u_{i}^{(m+1)} N_{i}\right]=0} \\
\left|T_{i} \sigma_{i j}^{(m)} N_{j}\right|=-\mu_{f} N_{i} \sigma_{i j}^{(m)} N_{j}
\end{gathered}
$$

while the thermal conditions (7) lead to

$$
\begin{gathered}
{\left[q_{j}^{(-1)} N_{j}\right]=\mu_{f} N_{i} \sigma_{i j}^{(-1)} N_{j}\left[\frac{\partial u_{m}^{(0)}}{\partial t} T_{m}\right] ; \quad\left[T^{(0)}\right]=0} \\
{\left[q_{j}^{(0)} N_{j}\right]=\mu_{f}\left(N_{i} \sigma_{i j}^{(-1)} N_{j}\left[\frac{\partial u_{m}^{(1)}}{\partial t} T_{m}\right]+N_{i} \sigma_{i j}^{(0)} N_{j}\left[\frac{\partial u_{m}^{(0)}}{\partial t} T_{m}\right]\right) ;} \\
{\left[T^{(1)}\right]=0} \\
{\left[q_{j}^{(1)} N_{j}\right]=\mu_{f}\left(N_{i} \sigma_{i j}^{(-1)} N_{j}\left[\frac{\partial u_{m}^{(2)}}{\partial t} T_{m}\right]+N_{i} \sigma_{i j}^{(0)} N_{j}\left[\frac{\partial u_{m}^{(1)}}{\partial t} T_{m}\right]\right)} \\
+\mu_{f}\left(N_{i} \sigma_{i j}^{(1)} N_{j}\left[\frac{\partial u_{m}^{(0)}}{\partial t} T_{m}\right]\right) ; \quad\left[T^{(2)}\right]=0
\end{gathered}
$$

The development of the crack-tip heat flow (8) is calculated, using the link between the length elements in the physical and reference cells $d s=\varepsilon d s_{y}$, as :

$$
D^{\varepsilon}=D^{(0)}+\varepsilon D^{(1)}+\varepsilon^{2} D^{(2)}+\ldots
$$

The expressions of the terms $D^{(i)}$ with $i=0,1,2$ in the development are presented in the Appendix A. 
As concerns the energy release rate (10) it can be developed in the form :

$$
\mathcal{G}^{d \varepsilon}=\frac{1}{\varepsilon} \mathcal{G}^{(-1)}+\mathcal{G}^{(0)}+\varepsilon \mathcal{G}^{(1)}+\ldots
$$

where the explicit expressions of $\mathcal{G}^{(i)}$ with $i=0,1,2$ are also presented in the Appendix A.

Introduction of the expansions of $\mathcal{G}^{d \varepsilon}$ and $D^{\varepsilon}$ in the energy relation (9) provides the link between different orders terms of heat dissipation and energy-release rate :

$$
D^{(0)}=\frac{1}{2} \frac{d d}{d t} L_{c} \mathcal{G}^{(-1)} \quad ; \quad D^{(1)}=\frac{1}{2} \frac{d d}{d t} L_{c} \mathcal{G}^{(0)} \quad ; \quad D^{(2)}=\frac{1}{2} \frac{d d}{d t} L_{c} \mathcal{G}^{(1)}
$$

From Equations (18), (21), (22), (23) and (28), we deduce at the order $\varepsilon^{-2}$ the problem for $\mathbf{u}^{(\mathbf{0})}$ and $T^{(0)}$ given in the Appendix A, equations $(70-72)$ and $(73-75)$. The solution of this problem is obtained by choosing the macroscopic displacements and temperature fields $\mathbf{u}^{(\mathbf{0})}(\mathbf{x}, \mathbf{t})$ and $T^{(0)}(\mathbf{x}, \mathbf{t})$, independent of the microscopic variables $y_{i}$.

At the order $\varepsilon^{-1}$, from (19), (21), (22), (24) and (28) we get the problems for $\mathbf{u}^{(1)}$ and $T^{(1)}$ in the form :

$$
\begin{gathered}
\frac{\partial}{\partial y_{j}}\left(a_{i j k l} e_{y k l}\left(\mathbf{u}^{(1)}\right)\right)=0 \quad \text { in } Y \\
{\left[\left(a_{i j k l}\left(e_{x k l}\left(\mathbf{u}^{(0)}\right)+e_{y k l}\left(\mathbf{u}^{(1)}\right)\right)-b_{i j}\left(T^{(0)}-T_{0}\right)\right) N_{j}\right]=0} \\
N_{i}\left(a_{i j k l}\left(e_{x k l}\left(\mathbf{u}^{(0)}\right)+e_{y k l}\left(\mathbf{u}^{(1)}\right)\right)-b_{i j}\left(T^{(0)}-T_{0}\right)\right) N_{j}<0 \\
\left|T_{i}\left(a_{i j k l}\left(e_{x k l}\left(\mathbf{u}^{(0)}\right)+e_{y k l}\left(\mathbf{u}^{(1)}\right)\right)-b_{i j}\left(T^{(0)}-T_{0}\right)\right) N_{j}\right|= \\
-\mu_{f} N_{i}\left(a_{i j k l}\left(e_{x k l}\left(\mathbf{u}^{(0)}\right)+e_{y k l}\left(\mathbf{u}^{(1)}\right)\right)-b_{i j}\left(T^{(0)}-T_{0}\right)\right) N_{j} \\
{\left[u_{i}^{(1)} N_{i}\right]=0}
\end{gathered}
$$

on $C Y$ and

$$
\begin{gathered}
-k\left(\frac{\partial^{2} T^{(1)}}{\partial y_{1}^{2}}+\frac{\partial^{2} T^{(1)}}{\partial y_{2}^{2}}\right)=0 \quad \text { in } \quad Y \\
{\left[T^{(1)}\right]=0 ; \quad\left[-k \frac{\partial T^{(1)}}{\partial y_{j}} N_{j}\right]=-\left[-k \frac{\partial T^{(0)}}{\partial x_{j}} N_{j}\right] \quad \text { on } C Y} \\
\lim _{r \rightarrow 0} \int_{\Gamma Y_{r}}-k\left(\frac{\partial T^{(0)}}{\partial x_{j}}+\frac{\partial T^{(1)}}{\partial y_{j}}\right) n_{j} d s_{y}=\frac{1}{2} \frac{d d}{d t} L_{c} \mathcal{G}^{(0)} \text { at }\left(y_{1}, y_{2}\right)=\left( \pm \frac{d L_{c}}{2}, 0\right)
\end{gathered}
$$

On the external boundaries of $Y$, periodicity conditions complete the formulation of the cell problems.

Let us define a system of generators for the macroscopic strains $\mathbf{E}^{p q}$ that respect the contact boundary conditions. An example used in the simulations is given in the Appendix B. The macroscopic strains are obtained as linear combination of the elements of this system as :

$$
e_{x i j}\left(\mathbf{u}^{(0)}\right)=\varrho_{p q}\left(\mathbf{u}^{(0)}\right) E_{i j}^{p q}
$$


Based on the previous boundary-value problem for the first-order corrector $\mathbf{u}^{(1)}$ we deduce its general expression in the form :

$$
\mathbf{u}^{(1)}(\mathbf{x}, \mathbf{y}, t)=\boldsymbol{\xi}^{p q}(\mathbf{y}) \varrho_{p q}\left(\mathbf{u}^{(0)}\right)(\mathbf{x}, t)+\phi(\mathbf{y})\left(T^{(0)}(\mathbf{x}, t)-T_{0}\right)
$$

where $\boldsymbol{\xi}^{p q}$ and $\phi$ are respectively solutions of the cell problems (76 - 80) and (81 - 85) given in the Appendix B.

Taking into account the orientation of the microcrack, with $N_{1}=0$ and $N_{2}=1$, the solution of $(34-36)$ has the general form

$$
T^{(1)}=\theta(\mathbf{y}) \frac{\partial T^{(0)}}{\partial x_{2}}(\mathbf{x}, t)
$$

where $\theta$ is obtained by solving the cell problem $(86-88)$ in the Appendix B.

More details about the formulation of these unit-cell solutions and their compatibility with the contact conditions on the crack faces are given in the Appendix B.

At the order $\varepsilon^{0}$, we obtain for temperature and displacement fields :

$$
\begin{gathered}
c \frac{\partial T^{(0)}}{\partial t}+b_{i j} T^{(0)} \frac{\partial}{\partial t}\left(e_{x i j}\left(\mathbf{u}^{(0)}\right)+e_{y i j}\left(\mathbf{u}^{(1)}\right)\right)+\frac{\partial q_{j}^{(0)}}{\partial x_{j}}+\frac{\partial q_{j}^{(1)}}{\partial y_{j}}=0 \quad \text { in } Y \\
{\left[T^{(2)}\right]=0 ;\left[q_{j}^{(1)} N_{j}\right]=\mu_{f} N_{i} \sigma_{i j}^{(0)} N_{j}\left[\frac{\partial u_{m}^{(1)}}{\partial t} T_{m}\right] \quad \text { on } C Y} \\
\lim _{r \rightarrow 0} \int_{\Gamma Y_{r}} q_{j}^{(1)} n_{j} d s_{y}=\frac{1}{2} \frac{d d}{d t} L_{c} \mathcal{G}^{(1)} \quad \text { at }\left(y_{1}, y_{2}\right)=\left( \pm \frac{d L_{c}}{2}, 0\right)
\end{gathered}
$$

and

$$
\begin{gathered}
\rho \frac{\partial^{2} u_{i}^{(0)}}{\partial t^{2}}-\frac{\partial \sigma_{i j}^{(0)}}{\partial x_{j}}-\frac{\partial \sigma_{i j}^{(1)}}{\partial y_{j}}=0 \quad \text { in } Y \\
\left|T_{i} \sigma_{i j}^{(1)} N_{j}\right|=-\mu_{f} N_{i} \sigma_{i j}^{(1)} N_{j} \quad \text { on } C Y \\
{\left[\sigma_{i j}^{(1)} N_{j}\right]=0 ; \quad N_{i} \sigma_{i j}^{(1)} N_{j}<0 ; \quad\left[u_{i}^{(1)} N_{i}\right]=0 \quad \text { on } C Y}
\end{gathered}
$$

To obtain the macroscopic thermo-mechanical equations we apply the mean value operator $\langle\cdot\rangle=\frac{1}{|Y|} \int_{Y} \cdot d y$, with $|Y|={L_{c}}^{2}$, to (40) and (43). Integration by parts and use of the periodicity and boundary conditions on microcracks lead to :

$$
\begin{gathered}
\left(c+T^{(0)} S^{*}\right) \frac{\partial T^{(0)}}{\partial t}+T^{(0)} \beta_{i j} \frac{\partial e_{x i j}\left(\mathbf{u}^{(0)}\right)}{\partial t}+\frac{\partial Q_{j}^{(0)}}{\partial x_{j}}= \\
\frac{\mathcal{G}^{(1)}}{L_{c}} \frac{d d}{d t}+\frac{1}{L_{c}^{2}} \int_{C Y} \sigma_{i j}^{(0)} N_{j}\left[\dot{u}_{i}^{(1)}\right] d s_{y} \\
\rho^{e f f} \frac{\partial^{2} u_{i}^{(0)}}{\partial t^{2}}-\frac{\partial \Sigma_{i j}^{(0)}}{\partial x_{j}}=0
\end{gathered}
$$

with the macroscopic stress $\Sigma_{i j}^{(0)}=\left\langle\sigma_{i j}^{(0)}\right\rangle$ and heat flux $Q_{j}^{(0)}=\left\langle q_{j}^{(0)}\right\rangle$ :

$$
\begin{aligned}
\Sigma_{i j}^{(0)} & =C_{i j p q} e_{x p q}\left(\mathbf{u}^{(0)}\right)-\vartheta_{i j}\left(T^{(0)}-T_{0}\right) \\
Q_{i}^{(0)} & =-k_{i j}^{e f f} \frac{\partial T^{(0)}}{\partial x_{j}}
\end{aligned}
$$


The particular expressions of the effective moduli $S^{*}, C_{i j p q}, \rho^{e f f}, \vartheta_{i j}$ and $k_{i j}^{\text {eff }}$ are given in the Appendix B. They are calculated with the unit-cell solutions and depend on the normalized crack length $d$.

As concerns the source terms in the right member of the equation (46), their macroscopic expressions will be obtained in the next section. It is important to note that the first one is calculated as a limit at the microcrack tips and represents the heating due to the damage evolution. The second term is obtained as an integral on microcracks and expresses the frictional heating effect. When damage is not evolving only the second term will be non-vanishing, while for propagating microcracks without friction only the first term remains in (46).

\section{Dynamic damage law}

The asymptotic homogenization method is used here in combination with an energy analysis for microcracks propagating dynamically, in mode II, with frictional contact and heating conditions, in order to obtain a macroscopic damage law. A specific upscaling procedure, from microcracks to damage, has been proposed in previous studies $[15,53,30,16]$ and the objective of this section is to extend this approach to account for thermal effects in the case of dynamically propagating mode II microcracks with contact and friction.

In case of thermomechanical couplings, starting from the cell problems formulated in the previous section and following a similar procedure as in [15] and [53], we establish the following energy balance relation :

$$
\begin{array}{r}
\int_{C Y} \sigma_{i j}^{(0)} N_{j}\left[\dot{u}_{i}^{(1)}\right] d s_{y}-\frac{d d}{d t} L_{c} \mathcal{G}^{(1)}-\frac{1}{2} \frac{d}{d t} \int_{C Y} \sigma_{i j}^{(0)} N_{j}\left[u_{i}^{(1)}\right] d s_{y}= \\
\frac{1}{2} \frac{d}{d t} \int_{Y}\left(a_{i j k l} e_{x k l}\left(\mathbf{u}^{(0)}\right)-b_{i j}\left(T^{(0)}-T_{0}\right)\right) e_{y i j}\left(\mathbf{u}^{(1)}\right) d y \\
-\int_{Y}\left(a_{i j k l} e_{x k l}\left(\dot{\mathbf{u}}^{(0)}\right)-b_{i j} \dot{T}^{(0)}\right) e_{y i j}\left(\mathbf{u}^{(1)}\right) d y
\end{array}
$$

The proof of this identity is given in the Appendix C.

Based on the particular form of the microscopic first-order correctors (38) and (39) and using the frictional contact and heating boundary conditions, it is shown in the Appendix $\mathrm{C}$ that we can obtain from (50) the damage equation :

$$
\frac{d d}{d t}\left(\frac{\mathcal{G}^{(1)}}{L_{c}}-\mathcal{Y}+\mathcal{Y}_{f}\right)=0
$$

where the effective thermomechanical energy-release rate is

$$
\begin{gathered}
\mathcal{Y}=-\frac{1}{2} \frac{d C_{k l p q}}{d d} e_{x p q}\left(\mathbf{u}^{(0)}\right) e_{x k l}\left(\mathbf{u}^{(0)}\right) \\
+\frac{1}{2}\left(\frac{d \vartheta_{k l}}{d d}+\alpha \frac{d C_{p q k l}}{d d} \delta_{p q}\right)\left(T^{(0)}-T_{0}\right) e_{x k l}\left(\mathbf{u}^{(0)}\right)+\frac{1}{2} \frac{d S^{*}}{d d}\left(T^{(0)}-T_{0}\right)^{2}
\end{gathered}
$$

and the amount of energy dissipated by friction

$$
\mathcal{Y}_{f}=I_{m n p q} e_{x m n}\left(\mathbf{u}^{(0)}\right) e_{x p q}\left(\mathbf{u}^{(0)}\right)+J_{m n} e_{x m n}\left(\mathbf{u}^{(0)}\right)\left(T^{(0)}-T_{0}\right)-P\left(T^{(0)}-T_{0}\right)^{2}
$$


Explicit expressions of the effective coefficients $I_{m n p q}, J_{m n}$ and $P$ are given in the Appendix C.

The damage relation (51) can be viewed as an identity between the energy dissipated during the propagation of microcracks and the corresponding damage dissipation at the macroscopic scale, calculated with the difference between the total energy release rate and the dissipation due to frictional sliding.

The relation between the dynamic and the quasi-static energy release $[23,48]$ is $\mathcal{G}^{d \varepsilon}=g_{2}\left(v_{p}\right) \mathcal{G}^{\varepsilon}$, where $\mathcal{G}^{\varepsilon}=\varepsilon \mathcal{G}^{(1)}$ is the quasi-static energy release rate, $v_{p}=\frac{l_{c}}{2} \frac{d d}{d t}$ is the crack-tip speed and $g_{2}\left(v_{p}\right)$ a universal function for mode II propagation. Retaining the approximation proposed in [5] :

$$
g_{2}\left(v_{p}\right) \simeq \sqrt{1-\frac{v_{p}}{c_{R}}}
$$

we deduce

$$
\mathcal{G}^{(1)}=\frac{L_{c}}{l_{c}} \frac{\mathcal{G}^{d \varepsilon}}{\sqrt{1-\frac{l_{c}}{2 c_{R}} \frac{d d}{d t}}}
$$

If we adopt a Griffith type propagation criterion $\mathcal{G}^{d \varepsilon}=\mathcal{G}_{c}$, with $\mathcal{G}_{c}$ the fracture energy, then (51) leads to the damage law :

$$
\frac{d d}{d t}=\frac{2 C_{R}}{l_{c}}\left\langle 1-\frac{\mathcal{G}_{c}^{2}}{l_{c}^{2}\left(\mathcal{Y}-\mathcal{Y}_{f}\right)^{2}}\right\rangle
$$

where $\langle$.$\rangle represent the positive part.$

The experiments on the dynamic failure of some brittle materials reveal the necessity to consider velocity-dependent fracture energy. In the case of a linear approximation [16] : $\mathcal{G}_{c}\left(v_{p}\right)=\mathcal{G}_{c 0}\left(1+a . v_{p}\right)$ we obtain from (51) a more complex form of the damage law :

$$
\frac{d d}{d t}=\left\langle\frac{2 \Pi}{a^{2} \mathcal{G}_{c 0}^{2} l_{c}^{2}}-\frac{l_{c}}{a^{2} C_{R} \mathcal{G}_{c 0}^{2}}\left(\mathcal{Y}_{d}-\mathcal{Y}_{f}\right)^{2}-\frac{2}{a l_{c}}\right\rangle
$$

where

$$
\Pi=\sqrt{\left(a \mathcal{G}_{c 0}^{2} l_{c}+\frac{l_{c}^{3}}{2 C_{R}}\left(\mathcal{Y}-\mathcal{Y}_{f}\right)^{2}\right)^{2}-a^{2} \mathcal{G}_{c 0}^{2} l_{c}^{2}\left(\mathcal{G}_{c 0}^{2}-l_{c}^{2}\left(\mathcal{Y}_{d}-\mathcal{Y}_{f}\right)^{2}\right)}
$$

We remark that the microstructural length $l_{c}$ is present in the equations (56) and (57) and will give rise to size effects related related to damage evolution. Also, the energy-release rate terms depend on the macroscopic deformation and temperature assuring the thermoelastic and damage coupling. These effects will be illustrated by the response analysis considered in the last section.

At this point, using the developments in the Appendix C, we can deduce from (46) the macroscopic temperature equation in the form

$$
\left(c+T^{(0)} S^{*}\right) \frac{\partial T^{(0)}}{\partial t}+T^{(0)} \beta_{p q} \frac{\partial e_{x p q}\left(\mathbf{u}^{(0)}\right)}{\partial t}+\frac{\partial Q_{j}^{(0)}}{\partial x_{j}}=\mathcal{R}_{d}+\mathcal{R}_{f}
$$


The first term in the right member of this equation $\mathcal{R}_{d}$ represents a heat source related to damage evolution and is given by

$$
\mathcal{R}_{d}=\frac{d d}{d t}\left(\mathcal{Y}-\mathcal{Y}_{f}\right)=\frac{d d}{d t}\left(\frac{1}{2 l_{c}} \frac{2+a . l_{c} \frac{d d}{d t}}{\sqrt{1-\frac{l_{c}}{2 c_{R}} \frac{d d}{d t}}} \mathcal{G}_{c 0}\right)
$$

We can observe that the first expression of $\mathcal{R}_{d}$ is a typical damage dissipation formula with the energy-release rate $\mathcal{Y}-\mathcal{Y}_{f}$ involved in the previous damage laws. The heat source $\mathcal{R}_{d}$ corresponds to the first term in the right member of the temperature equation (46) and is activated in the material points where damage evolves.

The second term accounts for the frictional heating on microcracks and reads :

$$
\begin{gathered}
\mathcal{R}_{f}=\frac{d d}{d t}\left(I_{m n p q}^{2} e_{x m n}\left(\mathbf{u}^{(0)}\right) e_{x p q}\left(\mathbf{u}^{(0)}\right)+J_{m n}^{2} e_{x m n}\left(\mathbf{u}^{(0)}\right)\left(T^{(0)}-T_{0}\right)-P^{2}\left(T^{(0)}-T_{0}\right)^{2}\right) \\
+\mathcal{Z}_{m n p q}^{1} e_{x m n}\left(\mathbf{u}^{(0}\right) \frac{\partial e_{x p q}\left(\mathbf{u}^{(0)}\right)}{\partial t}+\mathcal{Z}_{p q}^{2} \frac{\partial e_{x p q}\left(\mathbf{u}^{(0)}\right)}{\partial t}\left(T^{(0)}-T_{0}\right) \\
+\mathcal{Z}_{m n}^{3} e_{x m n}\left(\mathbf{u}^{(0)}\right) \frac{\partial T^{(0)}}{\partial t}+\mathcal{Z}^{4}\left(T^{(0)}-T_{0}\right) \frac{\partial T^{(0)}}{\partial t}
\end{gathered}
$$

The details concerning the coefficients $\mathcal{Z}_{m n p q}^{1}, \mathcal{Z}_{m n}^{2}, \mathcal{Z}_{m n}^{3}, I_{m n p q}^{2}, J_{m n}^{2}$ and $P^{2}$ are given in the Appendix C. In the case of non-frictional sliding on microcracks, all of them are vanishing.

\section{Local macroscopic response}

The analysis of the local macroscopic response predicted by the new damage model is presented in this section. We first present the effective coefficients and their dependence on damage and friction coefficients, then we study the local macroscopic response. The coefficients are computed with unit-cell FE solutions for different microcrack lengths. For the study of the macroscopic response the solution of the damage law (57) coupled with the equation of temperature (59) in the adiabatic approximation is obtained for a given history of compression and shear macroscopic strains. Shear stresses are obtained from the constitutive law (48). Effects of the size of microstructure, shear strain rate, initial microcrack length, compression loading and friction coefficient will be discussed.

The generic material parameters used in the simulations for the PMMA polymer are summarized in Table 1 . A velocity-dependent fracture energy $\mathcal{G}_{c}\left(v_{p}\right)=$ $\mathcal{G}_{c 0}\left(1+a . v_{p}\right)$ is assumed for the mode II propagation.

\begin{tabular}{cccccccc}
\hline$E(\mathrm{GPa})$ & $\nu$ & $\rho\left(\mathrm{kg} \mathrm{m}^{-3}\right)$ & $c\left(\mathrm{Jm}^{-3} \mathrm{~K}^{-1}\right)$ & $\alpha\left(\mathrm{K}^{-1}\right)$ & $\mu_{f}$ & $\mathcal{G}_{c 0}\left(\mathrm{Jm}^{-2}\right)$ & $a\left(\mathrm{~s} \mathrm{~m}^{-1}\right)$ \\
\hline 5.6 & 0.33 & 1180 & $10^{6}$ & $10^{-4}$ & 0.5 & 2100 & 0.01 \\
\hline
\end{tabular}

TABLE 1: Material parameters for the PMMA polymer. 


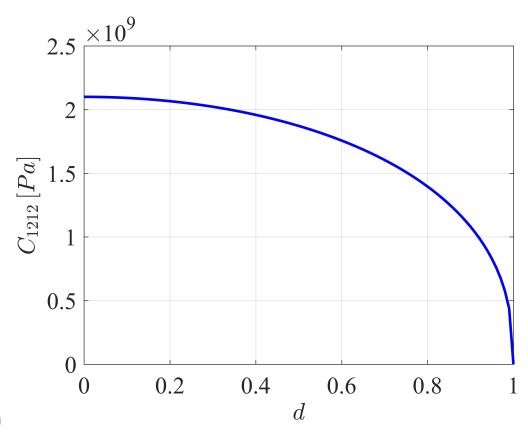

a)

Figure 3: Effective elastic coefficients $C_{1212}$ (a) and $C_{1222}$ (b) vs damage variable $d$ computed for the friction coefficients $\mu_{f}=0,0.25,0.5$.

\subsection{Homogenized coefficients}

All the effective coefficients were computed with the FE solution of the corresponding cell problems using the FEM software COMSOL Multiphysics. Since they are functions of damage, such computations have been done for different values of $d \in[0,1]$ and the results have been interpolated with polynomials.

For the elastic coefficients $C_{i j p q}$, the problem (76-80) is solved numerically on the unit cell. Except $C_{1222}$ and $C_{1212}$, the others components of the tensor $C_{i j p q}$ are not dependent on the damage variable. If $\lambda$ and $\mu$ are the Lamé coefficients, we obtain $C_{1111}=C_{2222}=\lambda+2 \mu, C_{1122}=C_{2211}=\lambda$ and $C_{1112}=C_{1211}=0$.

In Figure 3, the coefficients $C_{1212}$ and $C_{1222}$ are represented as functions of the damage variable $d$ for three values of the friction coefficient $\mu_{f}=0,0.25,0.5$. Damage induced degradation is observed for $C_{1212}$ without dependence on the friction coefficient. It represents the effect of the shear deformation of the unit cell on the macroscopic shear stress and the presence of microcracks makes easier the deformation of the cell, reducing the level of resulting shear stress. For a completely fractured cell, no shear stresses are generated since the two parts move freely. $C_{1222}$ characterizes the influence of the compression deformation $e_{x 22}$ on the macroscopic shear stress. In the absence of friction, there is no such influence while, in the case of frictional sliding, the compression influences the frictional resistance giving rise shear stresses. This effect is amplified by the value of the friction coefficient $\mu_{f}$ and also by the size of the friction zone, controlled by the normalized microcrack length $d$. Both effects can be seen in Figure 3b.

The effective thermoelastic coefficients $\vartheta_{i j}$ and the thermal modulus $S^{*}$ are given by the formulae (93) and, respectively, (89). The presence of microcracks with contact between their lips do not modify the initial values of the thermoelastic coefficients : $\vartheta_{i j}=b_{i j}$. The thermal contact conditions also preserves the initial value of the thermal modulus $S^{*}=0$.

As concerns the thermal conduction coefficients, they are calculated by (94) using the solution of the problem $(86-88)$. We remark that $\theta=0$ is the solution of this cell problem. The effective thermal conduction coefficients are not influenced by the presence of microcracks with contact conditions : $k_{11}^{e f f}=k_{22}^{e f f}=k$ and $k_{12}^{e f f}=0$. 


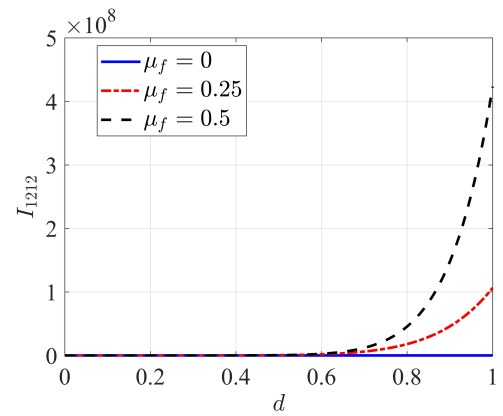

b)
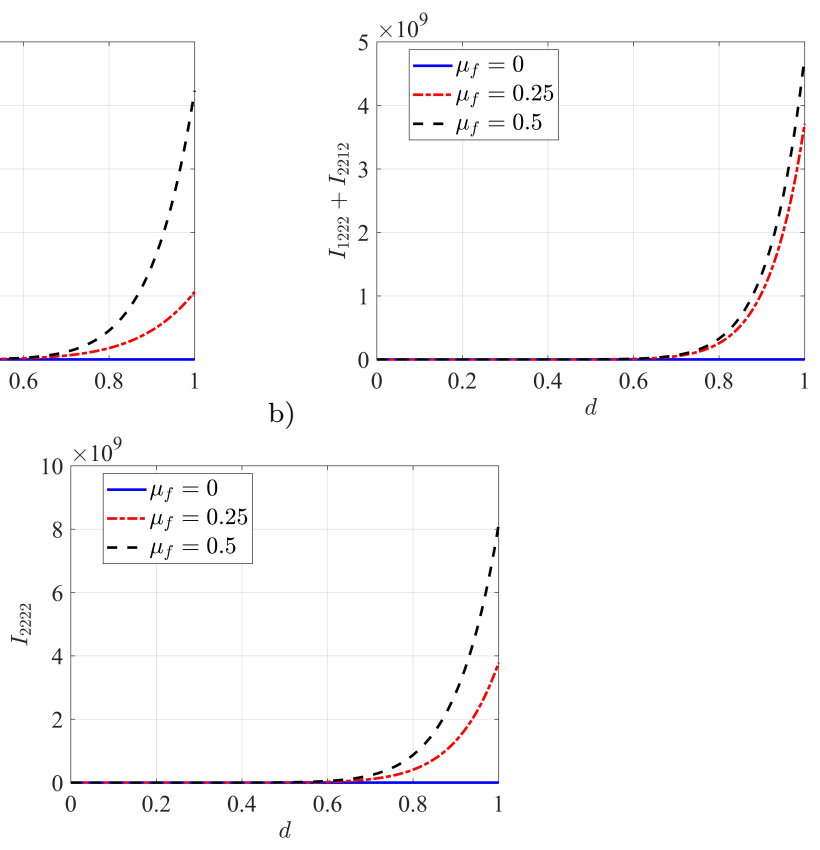

c)

Figure 4: Homogenized coefficients $I_{1212}$ (a), $I_{1222}+I_{2212}$ (b) and $I_{2222}$ (c) vs damage variable $d$, for $\mu_{f}=0,0.25,0.5$.

The components of the tensor $I_{m n p q}$ are controlling the amount of energy dissipated by friction (53) involved in the expression of the energy-release rate. They are evaluated numerically using the procedure described in the Appendix C. For the considered microcrack orientation and the continuity of the normal displacement field we deduce that the only non-vanishing components $I_{1212}, I_{1222}+$ $I_{2212}$ and $I_{2222}$. Figure 4 shows the effective coefficients $I_{1212}, I_{1222}+I_{2212}$, and $I_{2222}$ as functions of the damage variable $d$ for three values of $\mu_{f}$. As they influence the amount of energy dissipated by friction, these coefficients are increasing with the friction coefficient and the size of the friction zone which is proportional to $d$. If $\mu_{f}=0$ there is no dissipation and $I_{m n p q}=0$. The other coefficients $J_{m n}$ and $P$ involved in the expression (53) of $\mathcal{Y}_{f}$ are also vanishing. The thermal variations have no influence on the dissipated energy by frictional sliding.

Since only two strain components are used in the present analysis, the coefficients $\mathcal{Z}_{1212}^{1}$ and $\mathcal{Z}_{2212}^{1}$ of (61) are needed. They are represented in Figure 5 as functions of the damage variable $d$. These coefficients increase with the microcrack length and with the friction coefficient $\mu_{f}$. Since they characterize the effect of the strains and strain-rate on the frictional heating, larger microcracks or higher values of $\mu_{f}$ give rise to more heating at the macroscopic scale. The other coefficients $\mathcal{Z}_{m n}^{2}, \mathcal{Z}_{m n}^{3}$ and $\mathcal{Z}^{4}$ are equal to 0 . As for the terms in $\mathcal{Y}_{f}$, thermal variations do no influence on the energy dissipated as heat during frictional sliding on microcracks.

As concerns $I_{m n p q}^{2}$, the components $I_{1212}^{2}, I_{1222}^{2}+I_{2212}^{2}$ and $I_{2222}^{2}$ are represented in the Figure 6. They are also involved in the expression (61) of the frictional 

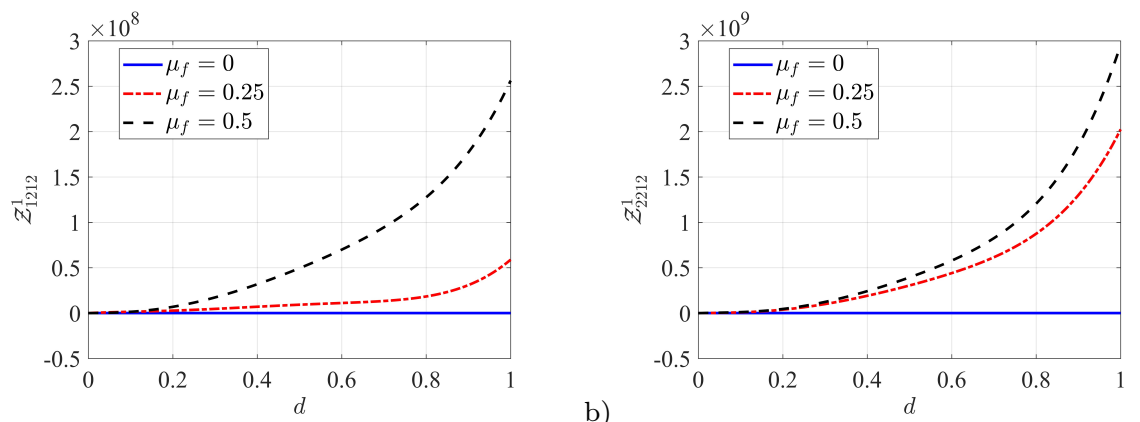

Figure 5: Coefficients $\mathcal{Z}_{1212}^{1}$ (a), $\mathcal{Z}_{2212}^{1}$ (b) vs damage variable $d$, for $\mu_{f}=$ $0,0.25,0.5$.

heat sources. All of them are increasing functions of $d$ and $\mu_{f}$ for the same physical reasons, while $J_{m n}^{2}$ and $P^{2}$ are vanishing.

a)

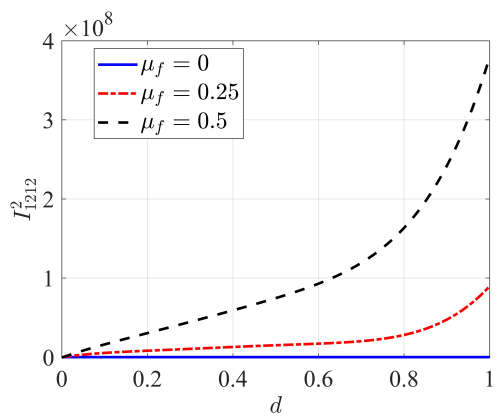

b)

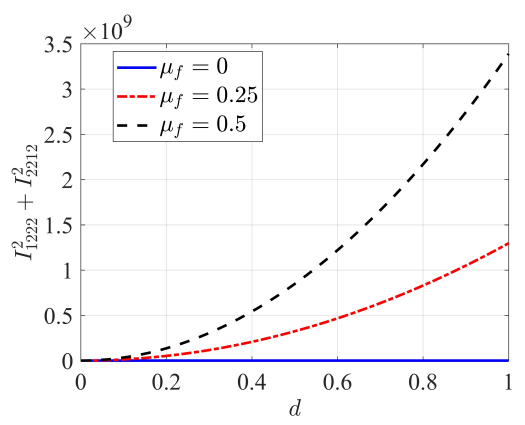

c)

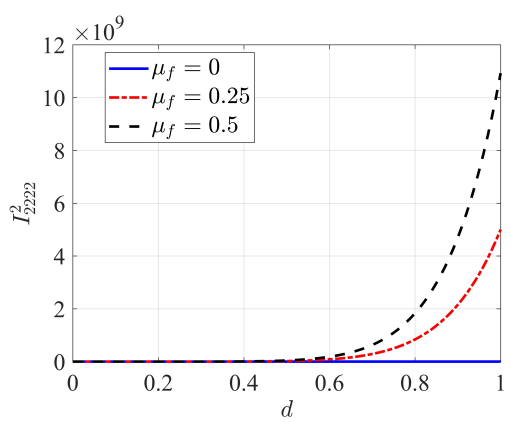

Figure 6: Components $I_{1212}^{2}$ (a), $I_{1222}^{2}+I_{2212}^{2}$ (b), $I_{2222}^{2}$ (c) of $I_{m n p q}^{2}$ vs damage variable $d$, for $\mu_{f}=0,0.25,0.5$. 

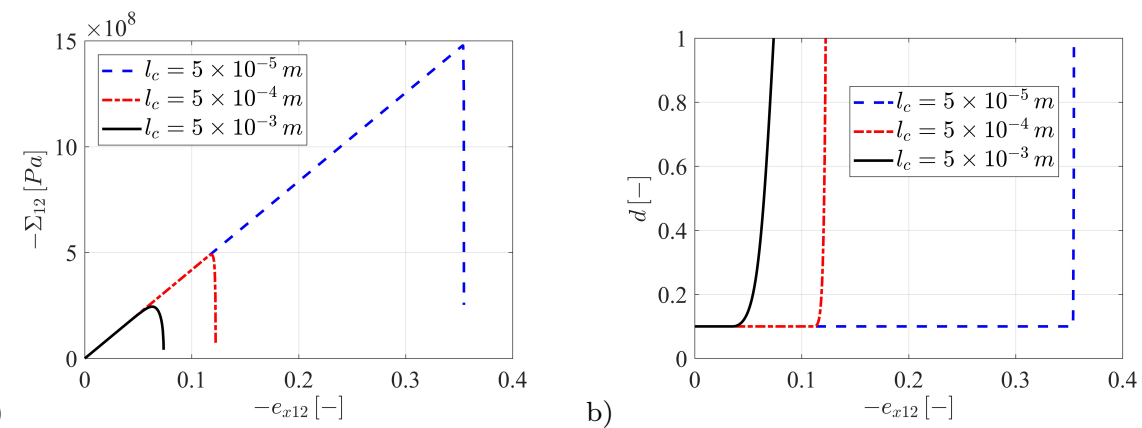

b)

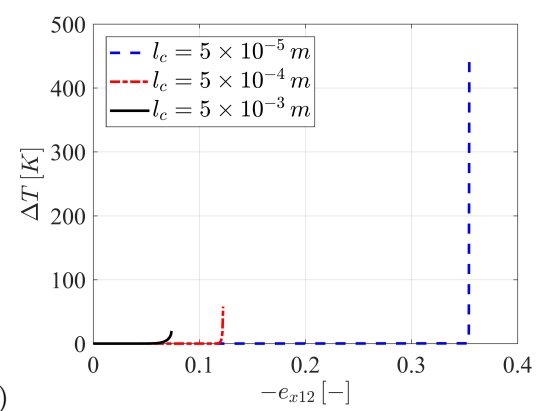

FiguRE 7: Effective stress $\Sigma_{12}$, damage variable $d$ and temperature variation $\Delta T$ vs. the strain $e_{x 12}$, for different values of the microstructural length : $l_{c}=5 \times$ $10^{-5} \mathrm{~m}, l_{c}=5 \times 10^{-4} \mathrm{~m}, l_{c}=5 \times 10^{-3} \mathrm{~m}$.

\subsection{Macroscopic response}

In order to analyze the macroscopic thermomechanical and damage response, a shear strain $e_{x 12}(t)=\dot{e}_{x 12} \times t$ with constant strain rate $\dot{e}_{x 12}$ and a constant compressive strain $e_{x 22}$ are assumed. Their numerical values are given in Table 2 , together with those for the microstructural size $l_{c}$, initial damage $d_{0}$ and initial temperature $T_{0}$. Unless specified, these generic values and the material parameters in Table 1 will be used in all the studies of the present section. The coupled system of the adiabatic temperature equation and the damage law is solved numerically and the macroscopic stresses are obtained with the computed damage and temperature solutions.

\begin{tabular}{ccccc}
\hline$e_{x 22}$ & $\dot{e}_{x 12}\left(\mathrm{~s}^{-1}\right)$ & $l_{c}(\mathrm{~m})$ & $d_{0}$ & $T_{0}(\mathrm{~K})$ \\
\hline$-5 \times 10^{-3}$ & -8000 & $5 \times 10^{-4}$ & 0.1 & 295.15 \\
\hline
\end{tabular}

TABLE 2: Parameters used in the study of the macroscopic response.

In Figure 7 , we plot the stress $\Sigma_{12}$, the damage variable $d$ and the temperature variation $\Delta T$ as function of the strain $e_{x 12}$ for different values of the size of microstructure $l_{c}$. As for the mode I microcracks [16], smaller microstructures are 
a)
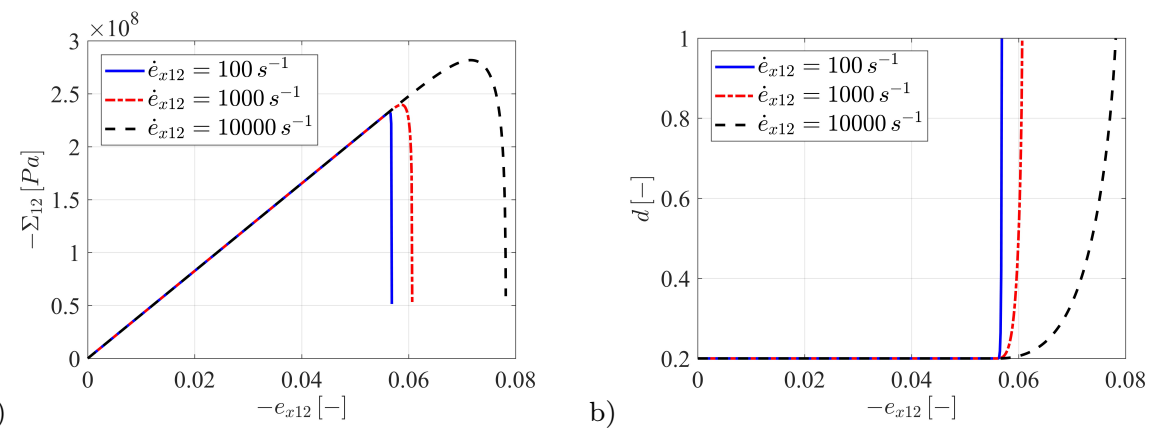

b)

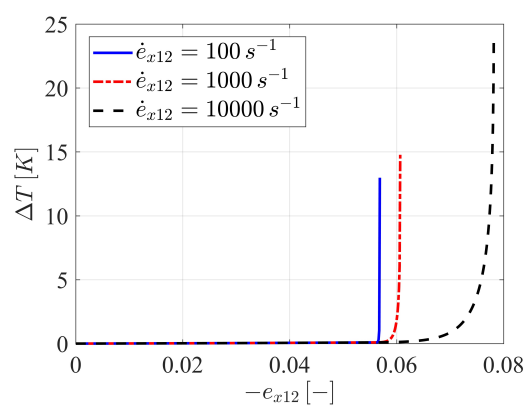

FiguRE 8: Effective stress $\Sigma_{12}$, damage variable $d$ and temperature variation $\Delta T$ vs. the strain $e_{x 12}$, for different values of strain rate : $\dot{e}_{x 12}=-100 s^{-1}, \dot{e}_{x 12}=$ $-1000 s^{-1}, \dot{e}_{x 12}=-10000 s^{-1}$.

more resistant to damage initiation since smaller microcracks are more difficult to propagate. Since larger microscopic cells have more important stored kinetic energy near the crack lips, the corresponding stress response is more ductile, with delayed damage evolution because a smaller amount of energy remains available for damage.

For small $l_{c}$, the damage propagates rapidly with more dissipated energy converted to heat. This leads to a more important temperature rise, while for slow evolutions of damage, corresponding to large $l_{c}$, we have less dissipation heating and the temperature increase is less important. The frictional heating effect is also present before damage initiation, but its value is negligible. Its contribution is increasing during damage evolution since $R_{f}$ depends on the damage rate.

Figure 8 shows the effect of the shear strain rate on damage, shear stress and temperature evolutions. A compression loading $e_{x 22}=-1 \times 10^{-2}$ is considered and the initial damage is $d_{0}=0.2$. For these computations we consider $l_{c}=1 \times 10^{-3}$ $\mathrm{m}$ as the size of the microstructure.

The strain-rate effect obtained for mode I microcracks $[30,16]$ is also present in this case. The increase of the loading rate induces more ductility in the macroscopic response, as a consequence of the inertial effects incorporated in the damage model. At high strain rates, a more important part of the supplied energy is stored as kinetic energy around microcrack tips, slowing the microcrack propagation. Compared to the case of opening microcracks $[30,16]$, where the kinetic energy 

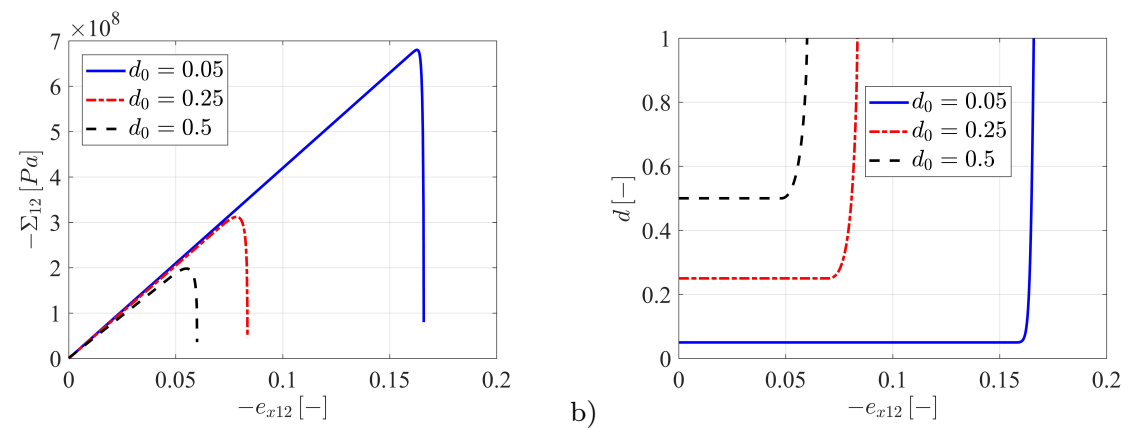

a)

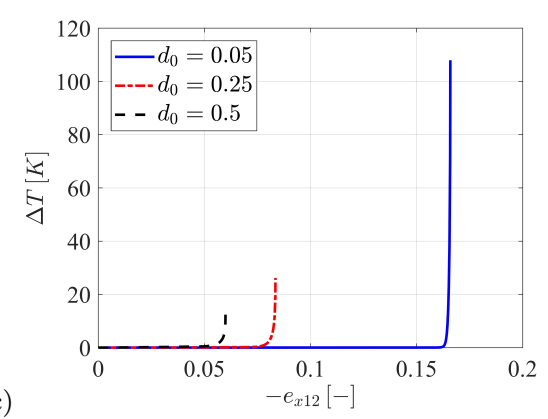

Figure 9: Effective stress $\Sigma_{12}$, damage variable $d$ and temperature variation $\Delta T$ vs. the strain $e_{x 12}$, for initial values of damage $d_{0}=0.05, d_{0}=0.2, d_{0}=0.5$.

may be more important, the strain-rate effect for closed and frictional microcracks is less pronounced.

Since the damage rate influences the amount of energy converted to heat, for higher loading rates the model predicts lower heat production during the propagation phase. This effects seems to be balanced by the duration of the heating process in the Figure $8 \mathrm{c}$. When the temperature variations are not so important (less than ten degrees in the figure) it is the second effect that dominates. For more radical temperature evolutions, like in the Figure 7, it is damage rate influence that prevails.

We study now the effects of the initial damage on the macroscopic response. The stress, temperature variation and the damage were computed for three different values of $d_{0}$ and represented in Figure 9. Retarded damage initiation leading to higher strength is observed for smaller values of $d_{0}$ and the strength decreases for higher values of $d_{0}$, as expected. This example is particularly interesting for the study of thermal effects. Larger initial crack lengths increase the frictional heating effect by augmenting the friction zone. Smaller initial lengths lead to high damage rates and corresponding crack propagation heating. The temperature behavior in Figure $9 \mathrm{c}$ shows that the second effect is dominant, since the temperature increase is substantially higher for smaller initial crack lengths.

Let us analyze now the effect of the compressive strain on the damage variable $d$, the macroscopic shear stress $\Sigma_{12}$ and the temperature variation $\Delta T$. Three values were considered for the compression loading : $e_{x 22}=-5 \times 10^{-3},-2 \times$ 

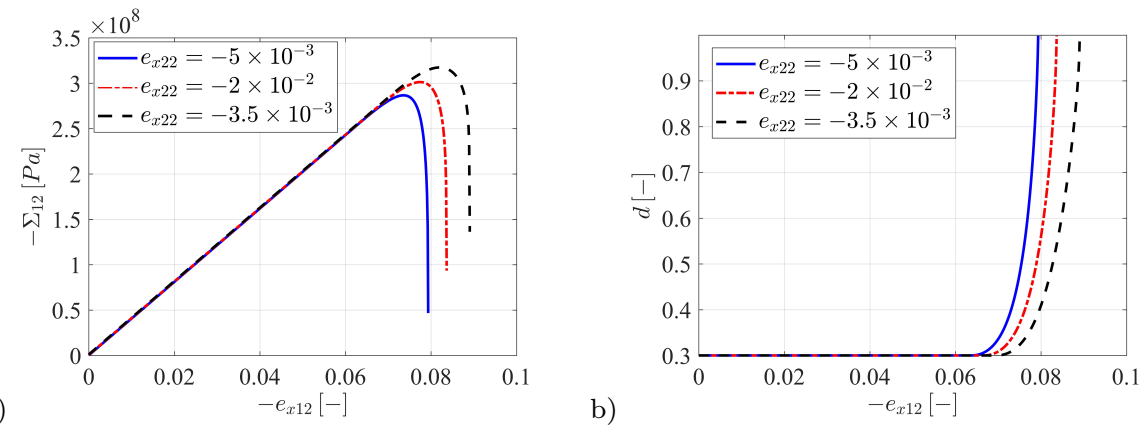

b)

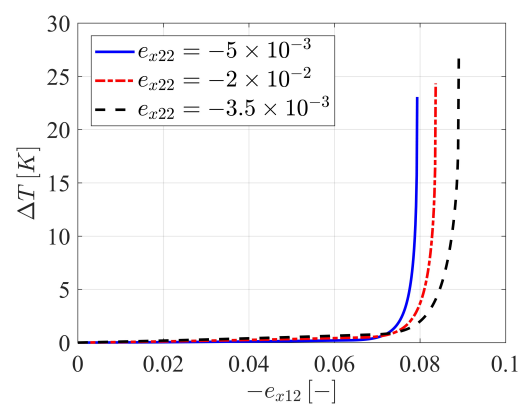

Figure 10: Effective stress $\Sigma_{12}$, damage variable $d$ and temperature variation $\Delta T$ vs. the strain $e_{x 12}$, for different compressive strains : $e_{x 22}=-1 \times 10^{-3}$, $e_{x 22}=-8 \times 10^{-3}, e_{x 22}=-1.5 \times 10^{-3}$.

$10^{-2},-3.5 \times 10^{-2}$. The shear strain rate used in this case is $\dot{e}_{x 12}=-10000 \mathrm{~s}^{-1}$ while $d_{0}=0.3$.

Figure 10 shows that the increase of the compressive strain retards the damage evolution due to the increased frictional resistance on microcracks. The corresponding frictional heating is also increased. Since the damage rates in the propagation phase are not radically different, the relatively small temperature increase could be explained as a frictional heating effect. Moreover, this effect is also present before damage initiation, as can be seen in the represented temperature evolution.

We finally consider the influence of friction coefficient $\mu_{f}$. In Figure 11, we plot the damage variable $d$, the stress $\Sigma_{12}$ and the temperature variation as function of the strain $e_{x 12}$, for different values of $\mu_{f}$. The compressive strain is $e_{x 22}=-3 \times$ $10^{-2}$ while the value of initial damage and microstructural size are correspondingly $d_{0}=0.25$, and $l_{c}=10^{-3} \mathrm{~m}$.

For large coefficients $\mu_{f}$, the frictional resistance is increased on microcracks and this induces larger shear strengths and delayed damage evolutions. This effects can be seen in Figure 11. As in the previous case, the temperature increase is slightly more important for large values of $\mu_{f}$ as a result of the frictional heating. Before damage initiation, a small increase of $\Delta T$ is present having the same frictional origin. Since the damage rates are close during the propagation phase, the comparison between the cases $\mu_{f}=0$ and $\mu_{f} \neq 0$ reveals the effect of frictional heating on microcracks. 

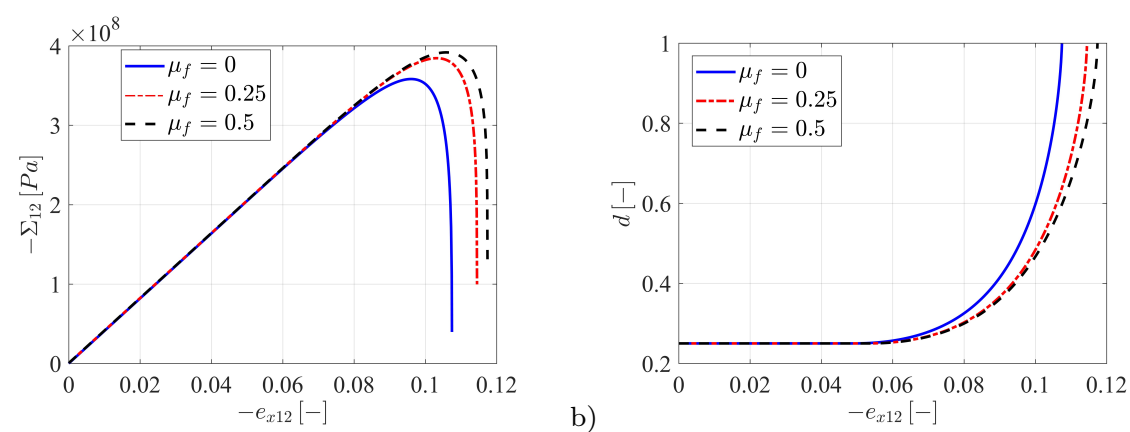

a)

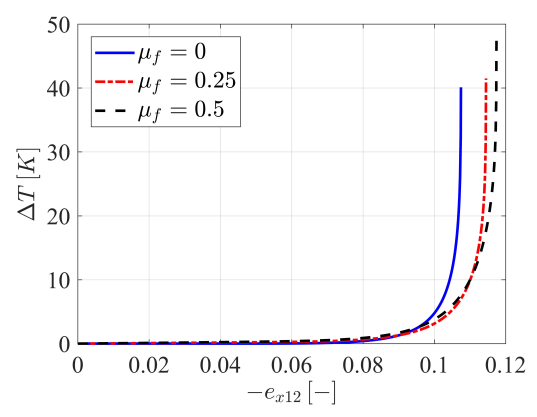

Figure 11: Effective stress $\Sigma_{12}$, damage variable $d$ and temperature variation $\Delta T$ for different values of the friction coefficient $\mu_{f}$.

\section{Numerical simulations of heating in mode II failure under impact}

We present in this section an example of application of the new model to simulate the mode II dynamic failure of a grooved PMMA panel under impact. The temperature evolution obtained in the FEM computations is compared to the experimental results presented in [12].

The details of the panel geometry and loading [12] are shown on Figure 12. The specimen has width $W=127 \mathrm{~mm}$, height $2 H=292 \mathrm{~mm}$ and thickness $T=8.5 \mathrm{~mm}$. Both sides of the panel have a groove of height $H_{g}=3.2 \mathrm{~mm}$ and depth $2.5 \mathrm{~mm}$. A starter notch of length $l=25 \mathrm{~mm}$ and width $1 \mathrm{~mm}$ is cut into one edge of the panel, inside the groove. The impact velocity $V_{i m p}=40 \mathrm{~m} \mathrm{~s}^{-1}$ is applied asymmetrically over a zone of $D=38 \mathrm{~mm}$, as shown in Fig. 12. Under this loading, a shear crack is initiated at the notch tip and propagates in the groove.

The model parameters used in the simulations are reported in Table 3. As in the previous section, we consider crack-speed dependency of the critical fracture energy in the form $\mathcal{G}_{c}=\mathcal{G}_{c 0}\left(1+a \cdot v_{p}\right)$.

\begin{tabular}{ccccccccc}
\hline$E(\mathrm{GPa})$ & $\nu$ & $\rho\left(\mathrm{kg} \mathrm{m}^{-3}\right)$ & $\alpha\left(\mathrm{K}^{-1}\right)$ & $c\left(\mathrm{~J} \mathrm{~m}^{-3} \mathrm{~K}^{-1}\right)$ & $\mu_{f}$ & $l_{c}(\mathrm{~m})$ & $\mathcal{G}_{c 0}\left(\mathrm{~J} \mathrm{~m}^{-2}\right)$ & $a\left(\mathrm{~s} \mathrm{~m}^{-1}\right)$ \\
\hline 5.5 & 0.36 & 1190 & $7 \times 10^{-5}$ & $1.725 \times 10^{6}$ & 0.5 & $3 \times 10^{-4}$ & 1900 & $5 \times 10^{-3}$ \\
\hline
\end{tabular}

TABLE 3: Parameters used in the FEM simulations. 

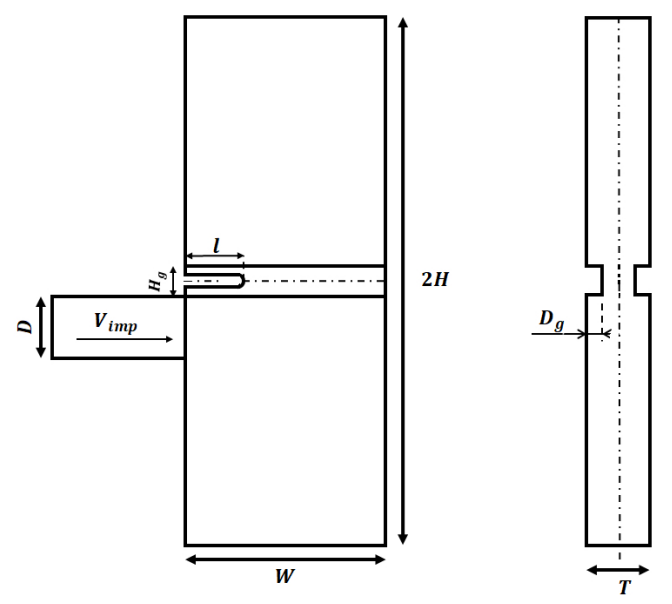

Figure 12: Geometry of the specimen and loading for mode II impact test.

In order to reproduce numerically the dynamic shear fracture of the specimen and the corresponding temperature evolution, we implemented the thermoelastic damage model in ABAQUS Explicit software [1]. The numerical integration of the temperature equation and of the damage evolution law is implemented in a VUMAT user subroutine for dynamic explicit analysis. On a time step and for a strain increment, an Euler forward scheme is used to solve the damage equation (57) at each integration point of the FE mesh, with the temperature at the end of the previous step. Then, a similar time integration scheme is employed to solve the adiabatic temperature equation (59) using the computed damage variable. The new values of the damage and temperature are used to update the stress components at the end of the time step according to the constitutive relation (48). Only the in-plane components of the strain are considered for the calculation of the damage energy $\mathcal{Y}-\mathcal{Y}_{f}$.

A 3D numerical modeling of the impact test is adopted in order to take into account the effect of the groove in the initiation and the evolution of the shear failure. The structure is then meshed with four nodes tetrahedral linear elements C3D4 with one integration point. The mesh is refined in the groove where the path of crack is expected, using elements of size $5 \times 10^{-2} \mathrm{~mm}$. Details of the mesh are shown on Figure 13. Element deletion is activated when the damage variable reaches a value of 0.99 . Unilateral contact with friction is introduced between the new formed interior faces after deletion.

The use of the damage model allows us to reproduce both fracture initiation at the notch tip and fracture propagation in the groove. A relatively straight trajectory is observed for the evolution of the damage band as depicted on Figure 14. The thickness of the damage band is about 3 times the element size. As in the purely mechanical case considered in [5], where a similar impact test [39] was reproduced numerically, the front velocity is increasing up to values close to the Rayleigh wave speed.

We focus here on the analysis of the thermal evolution during failure. In the experiment performed by Bjerke and Lambros [12], a vertical array of infrared 


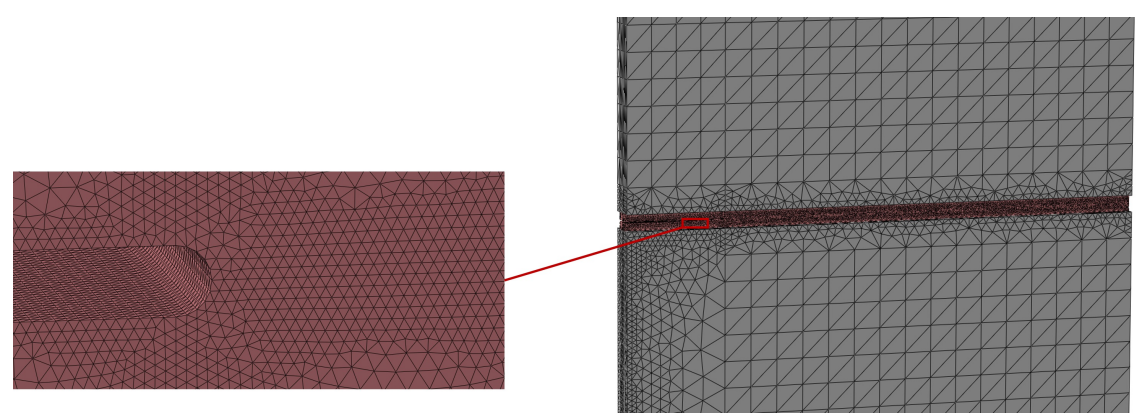

FiguRE 13: Details of the mesh used in the FE computations.

detectors has been placed in the groove, at $20 \mathrm{~mm}$ from the initial notch. These elements provide the temperature variations during the passage of the crack front. The computed temperature field around the damage band is represented in Figure 15. The area framed by the red rectangle is corresponding to the temperature detection points. The damage evolution in the elements is accompanied by a change in temperature.

In Figure 16a, we reproduce the measured temperature by Bjerke \& Lambros [12] using the array of infrared detectors located at $20 \mathrm{~mm}$ from the initial notch tip. The maximal temperature variation is obtained by the thermal signal of detector element 8 after an increase of about $85 K$, during a time interval between $55 \mu \mathrm{s}$ and $65 \mu \mathrm{s}$. From the numerical simulations, we obtained a relatively similar temperature increase of $85-90 \mathrm{~K}$ in the integration point of element located in the middle of the damage band and at $20 \mathrm{~mm}$ of the notch tip, as shown on Figure 16b. The temperature increase in the simulations takes place in $10 \mu \mathrm{s}$ which is close to that in the experiments.

Also measured in [12] are the temperatures in the flanking detectors 7 and 9. The size of these photovoltaic elements is of $8 \times 10^{-2} \mathrm{~mm}$. In the numerical simulations, the size of the mesh elements is approximately $5 \times 10^{-2} \mathrm{~mm}$. Despite this small difference, for the sake of comparison we represented in Figure 16b the temperatures computed in the corresponding lower and upper elements as can be seen in Figure 15. We remark that the computed values are relatively close to those

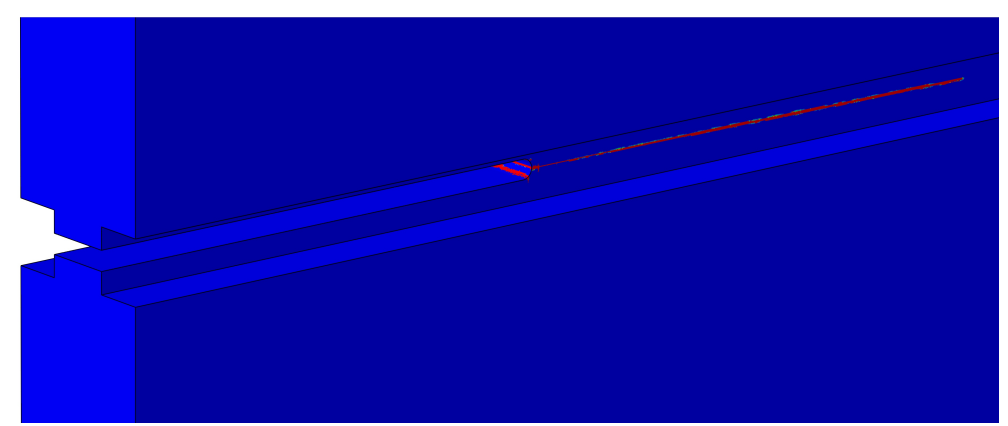

Figure 14: Damage band in the groove at $70 \mu \mathrm{s}$. 

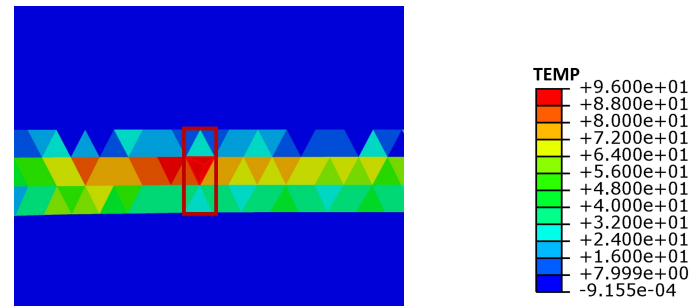

Figure 15: Temperature variation induced by damage at $20 \mathrm{~mm}$ of the initial notch tip.

measured by the corresponding detectors, with a mean value of about $30 \mathrm{~K}$ and a few $\mu s$ delay with respect to the temperature of the middle element. We finally note that the deletion of the damaged elements do not allow for the representation of further decrease of the temperature after the passage of the damage front.

While further studies are necessary to better understand the prediction potential of the new thermo-mechanical damage model, the first example presented in this section illustrated its capacity of reproducing the temperature variations associated to the dynamic shear failure of brittle solids.

\section{Conclusions}

We developed a coupled thermomechanical damage model for dynamic shear failure in brittle materials. The new damage law has been obtained using an adapted asymptotic homogenization procedure from the microscopic Griffith-type propagation criterion in mode II. Besides the unilateral contact and friction conditions, heat sources at the tips and over the lips of microcracks have been assumed in order to account for damage and frictional heating effects. The upscaling procedure led to a macroscopic equation of temperature with distributed heat sources related to damage propagation and frictional sliding mechanisms. A strong cou-
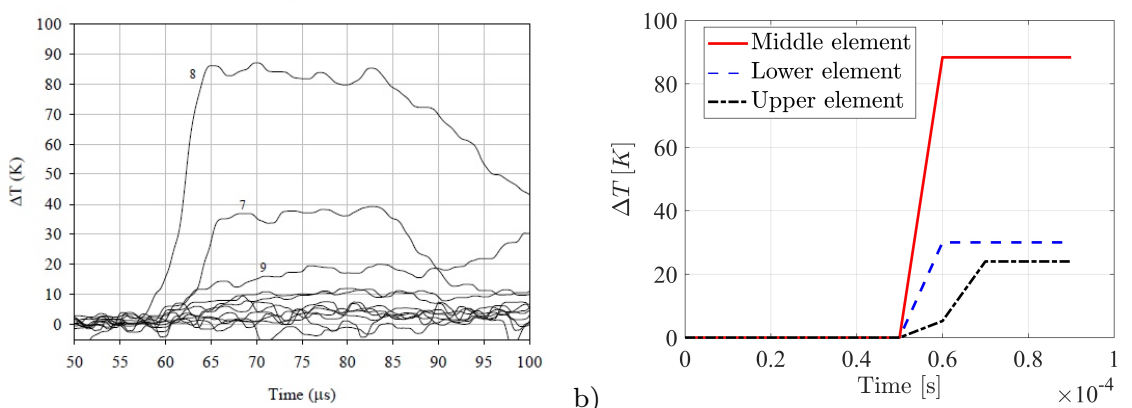

Figure 16: Temperature variation : measured values (a) from [12] and (b) computed values in 3 neighbor elements located in the region of IR detectors. 
pling between the mechanical, thermal and damage fields has been obtained. The analysis of the local macroscopic response predicted by the new model revealed specific influences of the microstructural size, the strain rate, the compression loading, the initial microcrack length and the friction coefficient. Finally, results of numerical simulations for the evolution of temperature during dynamic shear failure in PMMA samples under impact are presented. It was found that the model predictions are in good agreement with the experimental data.

\section{Appendix A : Asymptotic expansions}

The terms of orders $\varepsilon^{0}, \varepsilon^{1}, \varepsilon^{2}$ in the asymptotic expansions of the thermomechanical fields are given below. In the expansion of $\boldsymbol{q}^{\varepsilon}$ given in the equation (16), the terms $\boldsymbol{q}^{(p)}, p=0,1,2$ are :

$$
q_{j}^{(-1)}=-k \frac{\partial T^{(0)}}{\partial y_{j}} ; q_{j}^{(0)}=-k\left(\frac{\partial T^{(0)}}{\partial x_{j}}+\frac{\partial T^{(1)}}{\partial y_{j}}\right) ; q_{j}^{(1)}=-k\left(\frac{\partial T^{(1)}}{\partial x_{j}}+\frac{\partial T^{(2)}}{\partial y_{j}}\right)
$$

and the corresponding terms for $\boldsymbol{\sigma}^{\varepsilon}$ in the development (17) are :

$$
\begin{gathered}
\sigma_{i j}^{(-1)}=a_{i j k l} e_{y k l}\left(\mathbf{u}^{(0)}\right) \\
\sigma_{i j}^{(0)}=a_{i j k l}\left(e_{x k l}\left(\mathbf{u}^{(0)}\right)+e_{y k l}\left(\mathbf{u}^{(1)}\right)\right)-b_{i j}\left(T^{(0)}-T_{0}\right) \\
\sigma_{i j}^{(0)}=a_{i j k l}\left(e_{x k l}\left(\mathbf{u}^{(1)}\right)+e_{y k l}\left(\mathbf{u}^{(2)}\right)\right)-b_{i j} T^{(1)}
\end{gathered}
$$

For the expressions of different order terms $D^{(i)}$ in the asymptotic expansion (26) of the crack-tip heat flow $D^{\varepsilon}$ one obtains :

$$
\begin{gathered}
D^{(0)}=\lim _{r \rightarrow 0} \int_{\Gamma Y_{r}}-k \frac{\partial T^{(0)}}{\partial y_{j}} n_{j} d s_{y} ; D^{(1)}=\lim _{r \rightarrow 0} \int_{\Gamma Y_{r}}-k\left(\frac{\partial T^{(0)}}{\partial x_{j}}+\frac{\partial T^{(1)}}{\partial y_{j}}\right) n_{j} d s_{y} \\
D^{(2)}=\lim _{r \rightarrow 0} \int_{\Gamma Y_{r}}-k\left(\frac{\partial T^{(1)}}{\partial x_{j}}+\frac{\partial T^{(2)}}{\partial y_{j}}\right) n_{j} d s_{y}
\end{gathered}
$$

The terms of different orders in the asymptotic expansion (27) of the energyrelease rate $\mathcal{G}^{d \varepsilon}$ are :

$$
\begin{gathered}
\mathcal{G}^{(-1)}=\lim _{r \rightarrow 0} \int_{\Gamma Y_{r}}\left(\frac{1}{2} a_{i j k l} e_{y k l}\left(\mathbf{u}^{(0)}\right) e_{y i j}\left(\mathbf{u}^{(0)}\right) n_{1}-a_{i j k l} e_{y k l}\left(\mathbf{u}^{(0)}\right) n_{j} \frac{\partial u_{i}^{(0)}}{\partial y_{1}}\right) d s_{y}(67) \\
\mathcal{G}^{(0)}=\lim _{r \rightarrow 0} \int_{\Gamma Y_{r}}\left(\left(a_{i j k l} e_{y k l}\left(\mathbf{u}^{(0)}\right)\left(e_{x i j}\left(\mathbf{u}^{(0)}\right)+e_{y i j}\left(\mathbf{u}^{(1)}\right)\right)+b_{i j} T_{0} e_{y i j}\left(\mathbf{u}^{(0)}\right)\right) n_{1}\right. \\
\left.-a_{i j k l}\left(e_{x k l}\left(\mathbf{u}^{(0)}\right)+e_{y k l}\left(\mathbf{u}^{(1)}\right)\right) n_{j} \frac{\partial u_{i}^{(0)}}{\partial y_{1}}-a_{i j k l} e_{y k l}\left(\mathbf{u}^{(0)}\right) n_{j}\left(\frac{\partial u_{i}^{(0)}}{\partial x_{1}}+\frac{\partial u_{i}^{(1)}}{\partial y_{1}}\right)\right) d s_{y}
\end{gathered}
$$




$$
\begin{gathered}
\mathcal{G}^{(1)}=\lim _{r \rightarrow 0} \int_{\Gamma Y_{r}}\left(\left(\frac { 1 } { 2 } a _ { i j k l } \left(2 e_{y k l}\left(\mathbf{u}^{(0)}\right)\left(e_{x i j}\left(\mathbf{u}^{(0)}\right)+e_{y i j}\left(\mathbf{u}^{(1)}\right)\right)\right.\right.\right. \\
\left.+\left(e_{x i j}\left(\mathbf{u}^{(0)}\right)+e_{y i j}\left(\mathbf{u}^{(1)}\right)\right)\left(e_{x k l}\left(\mathbf{u}^{(0)}\right)+e_{y k l}\left(\mathbf{u}^{(1)}\right)\right)\right) \\
\left.+b_{i j} T_{0}\left(e_{x i j}\left(\mathbf{u}^{(0)}\right)+e_{y i j}\left(\mathbf{u}^{(1)}\right)\right)+c T^{(0)}+\frac{\rho}{8} \frac{\partial u_{i}^{(0)}}{\partial y_{1}} \frac{\partial u_{i}^{(0)}}{\partial y_{1}}\left(\frac{d d}{d t}\right)^{2}\right) n_{1} \\
-\left(a_{i j k l} e_{y k l}\left(\mathbf{u}^{(0)}\right) n_{j}\left(\frac{\partial u_{i}^{(1)}}{\partial x_{1}}+\frac{\partial u_{i}^{(2)}}{\partial y_{1}}\right)\right. \\
+\left(a_{i j k l}\left(e_{x k l}\left(\mathbf{u}^{(0)}\right)+e_{y k l}\left(\mathbf{u}^{(1)}\right)\right)-b_{i j}\left(T^{(0)}-T_{0}\right)\right) n_{j}\left(\frac{\partial u_{i}^{(0)}}{\partial x_{1}}+\frac{\partial u_{i}^{(1)}}{\partial y_{1}}\right) \\
\left.\left.+\left(a_{i j k l}\left(e_{x k l}\left(\mathbf{u}^{(1)}\right)+e_{y k l}\left(\mathbf{u}^{(2)}\right)\right)-b_{i j} T^{(1)}\right) n_{j} \frac{\partial u_{i}^{(0)}}{\partial y_{1}}\right)\right) d s_{y}
\end{gathered}
$$

For the velocity field the local relation $\frac{\partial u_{i}^{\varepsilon}}{\partial t} \simeq-\frac{d(l / 2)}{d t} \frac{\partial u_{i}^{\varepsilon}}{\partial x_{1}}=-\frac{\varepsilon L_{c}}{2} \frac{d d}{d t} \frac{\partial u_{i}^{\varepsilon}}{\partial x_{1}}$ in the vicinity of the crack tips $[23,7]$ has been used.

From Equations (18), (21), (22), (23) and (28), we deduce at the order $\varepsilon^{-2}$ the problems for $\mathbf{u}^{(\mathbf{0})}$ and $T^{(0)}$ as :

$$
\begin{gathered}
\frac{\partial}{\partial y_{j}}\left(a_{i j k l} e_{y k l}\left(\mathbf{u}^{(0)}\right)\right)=0 \quad \text { in } \\
{\left[a_{i j k l} e_{y k l}\left(\mathbf{u}^{(0)}\right) N_{j}\right]=0 ; \quad\left[u_{i}^{(0)} N_{i}\right]=0 ; \quad N_{i} a_{i j k l} e_{y k l}\left(\mathbf{u}^{(0)}\right) N_{j}<0} \\
\left|T_{i} a_{i j k l} e_{y k l}\left(\mathbf{u}^{(0)}\right) N_{j}\right|=-\mu_{f} N_{i} a_{i j k l} e_{y k l}\left(\mathbf{u}^{(0)}\right) N_{j}
\end{gathered}
$$

and

$$
\begin{gathered}
-k\left(\frac{\partial^{2} T^{(0)}}{\partial y_{1}^{2}}+\frac{\partial^{2} T^{(0)}}{\partial y_{2}^{2}}\right)=0 \quad \text { in } \\
{\left[T^{(0)}\right]=0 ; \quad\left[-k \frac{\partial T^{(0)}}{\partial y_{j}} N_{j}\right]=\mu_{f} N_{i} a_{i j k l} e_{y k l}\left(\mathbf{u}^{(0)}\right)\left[\frac{\partial u_{m}^{(0)}}{\partial t} T_{m}\right] N_{j}} \\
\lim _{r \rightarrow 0} \int_{\Gamma Y_{r}}-k \frac{\partial T^{(0)}}{\partial y_{j}} n_{j} d s_{y}=\frac{1}{2} \frac{d d}{d t} L_{c} \mathcal{G}^{(-1)} \text { at }\left(y_{1}, y_{2}\right)=\left( \pm \frac{d L_{c}}{2}, 0\right)
\end{gathered}
$$

The solution is obtained by choosing the macroscopic displacements and temperature fields $\mathbf{u}^{(\mathbf{0})}(\mathbf{x}, \mathbf{t})$ and $T^{(0)}(\mathbf{x}, \mathbf{t})$, independent of the microscopic variables $y_{i}$, which verify the systems $(70-72)$ and $(73-75)$.

Since $\mathbf{u}^{(0)}$ is not depending on $\mathbf{y}$, the expressions (67-68) show that $\mathcal{G}^{(-1)}$ and $\mathcal{G}^{(0)}$ are vanishing. As remarked in [16], the kinetic energy do not contribute to $\mathcal{G}^{(-1)}$ unless higher order correctors are considered for the thermomechanical fields.

\section{Appendix B : Computation of the effective coefficients}

We provide here the details concerning the computation of the homogenized coefficients appearing in the formulation of the macroscopic elasto-damage problem. 
The cell problem for $\boldsymbol{\xi}^{p q}$ is obtained in the form :

$$
\begin{gathered}
\frac{\partial}{\partial y_{j}}\left(a_{i j k l} e_{y k l}\left(\boldsymbol{\xi}^{p q}\right)\right)=0 \quad \text { in } Y \\
{\left[a_{i j k l} e_{y k l}\left(\boldsymbol{\xi}^{p q}\right) N_{j}\right]=-\left[a_{i j k l} E_{k l}^{p q} N_{j}\right]} \\
N_{i} a_{i j k l}\left(e_{y k l}\left(\boldsymbol{\xi}^{p q}\right)+E_{k l}^{p q}\right) N_{j}<0 \\
\left|T_{i} a_{i j k l}\left(e_{y k l}\left(\boldsymbol{\xi}^{p q}\right)+E_{k l}^{p q}\right) N_{j}\right|=-\mu_{f} N_{i} a_{i j k l}\left(e_{y k l}\left(\boldsymbol{\xi}^{p q}\right)+E_{k l}^{p q}\right) N_{j} \\
{\left[\xi_{i}^{p q} N_{i}\right]=0}
\end{gathered}
$$

The function $\phi$ is the solution of the boundary-value problem :

$$
\begin{gathered}
\frac{\partial}{\partial y_{j}}\left(a_{i j k l} e_{y k l}(\phi)\right)=0 \quad \text { in } \quad Y \\
{\left[a_{i j k l} e_{y k l}(\phi) N_{j}\right]=\left[b_{i j} N_{j}\right]} \\
N_{i}\left(a_{i j k l} e_{y k l}(\phi)-b_{i j}\right) N_{j}<0 \\
\left|T_{i}\left(a_{i j k l} e_{y k l}(\phi)-b_{i j}\right) N_{j}\right|=-\mu_{f} N_{i}\left(a_{i j k l} e_{y k l}(\phi)-b_{i j}\right) N_{j} \\
{\left[\phi_{i} N_{i}\right]=0}
\end{gathered}
$$

And for the function $\theta$ we obtain the cell problem :

$$
\begin{gathered}
-k\left(\frac{\partial^{2} \theta}{\partial y_{1}^{2}}+\frac{\partial^{2} \theta}{\partial y_{2}^{2}}\right)=0 \quad \text { in } Y \\
{\left[\frac{\partial \theta}{\partial y_{2}}\right]=0 ; \quad[\theta]=0 \quad \text { on } \quad C Y} \\
\lim _{r \rightarrow 0} \int_{\Gamma Y_{r}}-k\left(\frac{\partial \theta}{\partial y_{1}} n_{1}+\frac{\partial \theta}{\partial y_{2}} n_{2}\right) d s_{y}=0 \quad \text { at }\left(y_{1}, y_{2}\right)=\left( \pm \frac{d L_{c}}{2}, 0\right)
\end{gathered}
$$

As concerns the expressions of the homogenized coefficients, we obtain a thermal modulus $S^{*}$ given by the formula :

$$
S^{*}=\frac{1}{L_{c}^{2}} \int_{Y} b_{i j} e_{y i j}(\phi) d y
$$

The effective elastic moduli $C_{i j p q}^{\varrho}$ are computed with the cell solutions as :

$$
C_{i j p q}^{\varrho}=\frac{1}{L_{c}^{2}} \int_{Y} a_{i j k l}\left(E_{k l}^{p q}+e_{y k l}\left(\boldsymbol{\xi}^{p q}\right)\right) d y
$$

and the homogenized mass density :

$$
\rho^{e f f}=\frac{1}{|Y|} \int_{Y} \rho d y
$$

For the effective thermo-elastic coupling coefficients we obtain :

$$
\begin{aligned}
\beta_{p q}^{\varrho} & =\frac{1}{L_{c}^{2}} \int_{Y} b_{i j}\left(E_{i j}^{p q}+e_{y i j}\left(\boldsymbol{\xi}^{p q}\right)\right) d y \\
\vartheta_{i j} & =\frac{1}{L_{c}^{2}} \int_{Y}\left(b_{i j}-a_{i j k l} e_{y k l}(\boldsymbol{\phi})\right) d y
\end{aligned}
$$


The homogenized thermal conduction components are :

$$
\begin{gathered}
k_{11}^{e f f}=\frac{1}{|Y|} \int_{Y} k d y ; \quad k_{12}^{e f f}=\frac{1}{|Y|} \int_{Y} k \frac{\partial \theta}{\partial y_{1}} d y ; \\
k_{22}^{e f f}=\frac{1}{|Y|} \int_{Y} k\left(1+\frac{\partial \theta}{\partial y_{2}}\right) d y
\end{gathered}
$$

As explained in [53], a particular system of generators for the cell problem is necessary to respect the frictional contact conditions on the crack lips. The construction is specific to the present orientation of microcracks. The macroscopic strains $e_{x i j}\left(\mathbf{u}^{(0)}\right)$ are linear combinations of the elements of the system of macroscopic strains generators $\mathbf{E}^{p q}$ :

$$
e_{x i j}\left(\mathbf{u}^{(0)}\right)=\varrho_{p q}\left(\mathbf{u}^{(0)}\right) E_{i j}^{p q}
$$

where $\mathbf{E}^{p q}$ has the form :

$$
\mathbf{E}^{11}=\left(\begin{array}{cc}
-\frac{1}{\gamma} & 0 \\
0 & 0
\end{array}\right) \quad ; \mathbf{E}^{12}=\left(\begin{array}{cc}
0 & -\frac{1}{\gamma} \\
-\frac{1}{\gamma} & -\frac{1}{2 \gamma}
\end{array}\right) \quad ; \mathbf{E}^{22}=\left(\begin{array}{cc}
0 & -\frac{1}{\gamma} \\
-\frac{1}{\gamma} & -\frac{1}{\gamma}
\end{array}\right)
$$

and $\varrho_{p q}\left(\mathbf{u}^{(0)}\right)$ are defined as :

$$
\begin{gathered}
\varrho_{11}\left(\mathbf{u}^{(0)}\right)=-\gamma e_{x 11}\left(\mathbf{u}^{(0)}\right) ; \quad \varrho_{12}\left(\mathbf{u}^{(0)}\right)=\gamma\left(e_{x 22}\left(\mathbf{u}^{(0)}\right)-e_{x 12}\left(\mathbf{u}^{(0)}\right)\right) ; \\
\varrho_{22}\left(\mathbf{u}^{(0)}\right)=\gamma\left(e_{x 12}\left(\mathbf{u}^{(0)}\right)-2 e_{x 22}\left(\mathbf{u}^{(0)}\right)\right)
\end{gathered}
$$

In (46) the coefficients $\beta_{i j}$ are obtained as linear combination of $\beta_{i j}^{\varrho}$ using Equation (97) :

$$
\beta_{11}=-\gamma \beta_{11}^{\varrho} ; \quad \beta_{12}=\frac{\gamma}{2}\left(\beta_{22}^{\varrho}-2 \beta_{12}^{\varrho}\right) ; \quad \beta_{22}=2 \gamma\left(\beta_{12}^{\varrho}-\beta_{22}^{\varrho}\right)
$$

Using the equality $b_{i j}=\alpha a_{i j k l} \delta_{k l}$, we can write :

$$
\beta_{p q}^{\varrho}=\alpha C_{i j p q}^{\varrho} \delta_{i j}
$$

This gives :

$$
\begin{gathered}
\beta_{11}^{\varrho}=\alpha\left(C_{1111}^{\varrho}+C_{2211}^{\varrho}\right) ; \quad \beta_{12}^{\varrho}=\alpha\left(C_{1112}^{\varrho}+C_{2212}^{\varrho}\right) ; \\
\beta_{22}^{\varrho}=\alpha\left(C_{1122}^{\varrho}+C_{2222}^{\varrho}\right)
\end{gathered}
$$

Finally we obtain $\beta_{i j}$ as linear combination of $C_{i j p q}^{\varrho}$ :

$$
\begin{aligned}
& \beta_{11}=-\gamma \alpha\left(C_{1111}^{\varrho}+C_{2211}^{\varrho}\right) \\
& \beta_{12}=\frac{\gamma}{2} \alpha\left(C_{1122}^{\varrho}+C_{2222}^{\varrho}-2\left(C_{1112}^{\varrho}+C_{2212}^{\varrho}\right)\right) \\
& \beta_{22}=2 \gamma \alpha\left(C_{1112}^{\varrho}+C_{2212}^{\varrho}-C_{1122}^{\varrho}-C_{2222}^{\varrho}\right)
\end{aligned}
$$

The coefficients $C_{i j p q}$ in Equation (47) are linear combination of $C_{i j p q}^{\varrho}$ :

$$
\begin{gathered}
C_{i j 11}=-\gamma C_{i j 11}^{\varrho} ; \quad C_{i j 12}=\frac{\gamma}{2}\left(C_{i j 22}^{\varrho}-2 C_{i j 12}^{\varrho}\right) ; \\
C_{i j 22}=2 \gamma\left(C_{i j 12}^{\varrho}-C_{i j 22}^{\varrho}\right)
\end{gathered}
$$




\section{Appendix C : Construction of the damage law}

In this appendix we give details about the procedure used to obtain the damage law presented in Section 4.

We first prove the energy balance relation (50). Starting from similar cell problems as the one in Section 3 for the microscopic corrector $\mathbf{u}^{(1)}$, in $[15,53]$ the following energy balance has been established :

$$
\begin{aligned}
\frac{d}{d t} \int_{Y} \frac{1}{2} a_{i j k l} e_{y k l}\left(\mathbf{u}^{(1)}\right) e_{y i j} & \left(\mathbf{u}^{(1)}\right) d y+\frac{d d}{d t} L_{c} \mathcal{G}^{(1)} \\
& =\int_{C Y} a_{i j k l} e_{y k l}\left(\mathbf{u}^{(1)}\right) N_{j}\left[\dot{u}_{i}^{(1)}\right] d s_{y}
\end{aligned}
$$

The equivalent form of the equation $(70)$ :

$$
\frac{\partial}{\partial y_{j}}\left(a_{i j k l} e_{x k l}\left(\mathbf{u}^{(0)}\right)-b_{i j}\left(T^{(0)}-T_{0}\right)\right)=0
$$

multiplied by $\dot{u}_{i}^{(1)}$ and integrated over $Y$ leads to :

$$
\begin{gathered}
\int_{C Y}\left(a_{i j k l} e_{x k l}\left(\mathbf{u}^{(0)}\right)-b_{i j}\left(T^{(0)}-T_{0}\right)\right) N_{j}\left[\dot{u}_{i}^{(1)}\right] d s_{y}= \\
\int_{Y}\left(a_{i j k l} e_{x k l}\left(\mathbf{u}^{(0)}\right)-b_{i j}\left(T^{(0)}-T_{0}\right)\right) e_{y i j}\left(\dot{\mathbf{u}}^{(1)}\right) d y
\end{gathered}
$$

Combination of the relations (103) and (105) gives :

$$
\begin{gathered}
\int_{C Y}\left(a_{i j k l}\left(e_{x k l}\left(\mathbf{u}^{(0)}\right)+e_{y k l}\left(\mathbf{u}^{(1)}\right)\right)-b_{i j}\left(T^{(0)}-T_{0}\right)\right) N_{j}\left[\dot{u}_{i}^{(1)}\right] d s_{y} \\
=\frac{d d}{d t} L_{c} \mathcal{G}^{(1)}+\frac{d}{d t} \int_{Y} \frac{1}{2} a_{i j k l} e_{y k l}\left(\mathbf{u}^{(1)}\right) e_{y i j}\left(\mathbf{u}^{(1)}\right) d y \\
\quad+\int_{Y}\left(a_{i j k l} e_{x k l}\left(\mathbf{u}^{(0)}\right)-b_{i j}\left(T^{(0)}-T_{0}\right)\right) e_{y i j}\left(\dot{\mathbf{u}}^{(1)}\right) d y
\end{gathered}
$$

Using the Reynolds transport theorem [52] together with the singularity of the $\mathbf{u}^{(1)}$ field, for the last integral in (106) we get :

$$
\begin{gathered}
\int_{Y}\left(a_{i j k l} e_{x k l}\left(\mathbf{u}^{(0)}\right)-b_{i j}\left(T^{(0)}-T_{0}\right)\right) e_{y i j}\left(\dot{\mathbf{u}}^{(1)}\right) d y= \\
\frac{d}{d t} \int_{Y}\left(a_{i j k l} e_{x k l}\left(\mathbf{u}^{(0)}\right)-b_{i j}\left(T^{(0)}-T_{0}\right)\right) e_{y i j}\left(\mathbf{u}^{(1)}\right) d y \\
-\int_{Y}\left(a_{i j k l} e_{x k l}\left(\dot{\mathbf{u}}^{(0)}\right)-b_{i j} \dot{T}^{(0)}\right) e_{y i j}\left(\mathbf{u}^{(1)}\right) d y
\end{gathered}
$$

such that (106) becomes :

$$
\begin{gathered}
\int_{C Y}\left(a_{i j k l}\left(e_{x k l}\left(\mathbf{u}^{(0)}\right)+e_{y k l}\left(\mathbf{u}^{(1)}\right)\right)-b_{i j}\left(T^{(0)}-T_{0}\right)\right) N_{j}\left[\dot{u}_{i}^{(1)}\right] d s_{y}-\frac{d d}{d t} L_{c} \mathcal{G}^{(1)} \\
-\frac{1}{2} \frac{d}{d t} \int_{Y}\left(a_{i j k l}\left(e_{x k l}\left(\mathbf{u}^{(0)}\right)+e_{y k l}\left(\mathbf{u}^{(1)}\right)\right)-b_{i j}\left(T^{(0)}-T_{0}\right)\right) e_{y i j}\left(\mathbf{u}^{(1)}\right) d y \\
=\frac{1}{2} \frac{d}{d t} \int_{Y}\left(a_{i j k l} e_{x k l}\left(\mathbf{u}^{(0)}\right)-b_{i j}\left(T^{(0)}-T_{0}\right)\right) e_{y i j}\left(\mathbf{u}^{(1)}\right) d y \\
-\int_{Y}\left(a_{i j k l} e_{x k l}\left(\dot{\mathbf{u}}^{(0)}\right)-b_{i j} \dot{T}^{(0)}\right) e_{y i j}\left(\mathbf{u}^{(1)}\right) d y
\end{gathered}
$$


On the other hand, we can rewrite the equation (29) as :

$$
\frac{\partial}{\partial y_{j}}\left(a_{i j k l}\left(e_{x k l}\left(\mathbf{u}^{(0)}\right)+e_{y k l}\left(\mathbf{u}^{(1)}\right)\right)-b_{i j}\left(T^{(0)}-T_{0}\right)\right)=0
$$

Multiplication by $u_{i}^{(1)}$ and integration on $Y$ give :

$$
\begin{gathered}
\int_{Y}\left(a_{i j k l}\left(e_{x k l}\left(\mathbf{u}^{(0)}\right)+e_{y k l}\left(\mathbf{u}^{(1)}\right)\right)-b_{i j}\left(T^{(0)}-T_{0}\right)\right) e_{y i j}\left(\mathbf{u}^{(1)}\right) d y= \\
\int_{C Y}\left(a_{i j k l}\left(e_{x k l}\left(\mathbf{u}^{(0)}\right)+e_{y k l}\left(\mathbf{u}^{(1)}\right)\right)-b_{i j}\left(T^{(0)}-T_{0}\right)\right) N_{j}\left[u_{i}^{(1)}\right] d s_{y}(110)
\end{gathered}
$$

This identity can be used in (108) to obtain the energy relation (50).

Let us now prove the damage relations (51-53). From the expression of $\mathbf{u}^{(1)}$ in (38), we deduce the following relations :

$$
\begin{aligned}
\int_{Y} a_{i j k l} e_{y i j}\left(\mathbf{u}^{(1)}\right) d y & =\int_{Y} a_{i j k l} e_{y i j}\left(\boldsymbol{\xi}^{p q}\right) d y \varrho_{p q}\left(\mathbf{u}^{(0)}\right) \\
& +\int_{Y} a_{i j k l} e_{y i j}(\boldsymbol{\phi}) d y\left(T^{(0)}-T_{0}\right) \\
\int_{Y} b_{i j} e_{y i j}\left(\mathbf{u}^{(1)}\right) d y & =\int_{Y} b_{i j} e_{y i j}\left(\boldsymbol{\xi}^{p q}\right) d y \varrho_{p q}\left(\mathbf{u}^{(0)}\right) \\
& +\int_{Y} b_{i j} e_{y i j}(\boldsymbol{\phi}) d y\left(T^{(0)}-T_{0}\right)
\end{aligned}
$$

And using the expressions of the effective coefficients (90), (92), (93) and (89), we deduce :

$$
\begin{aligned}
\int_{Y} a_{i j k l} e_{y i j}\left(\boldsymbol{\xi}^{p q}\right) d y & =L_{c}^{2} C_{k l p q}^{\varrho}-a_{k l i j} E_{i j}^{p q} \\
\int_{Y} b_{i j} e_{y i j}\left(\boldsymbol{\xi}^{p q}\right) d y & =\alpha \delta_{k l}\left(L_{c}^{2} C_{k l p q}^{\varrho}-a_{k l i j} E_{i j}^{p q}\right) \\
\int_{Y} a_{i j k l} e_{y i j}(\phi) d y & =b_{k l}-L_{c}^{2} \vartheta_{k l} \\
\int_{Y} b_{i j} e_{y i j}(\phi) d y & =L_{c}^{2} S^{*}
\end{aligned}
$$

Replacing the equations (113-116) in (111-112) gives :

$$
\begin{gathered}
\int_{Y} a_{i j k l} e_{y i j}\left(\mathbf{u}^{(1)}\right) d y=\left(L_{c}^{2} C_{k l p q}^{\varrho}-a_{k l i j} E_{i j}^{p q}\right) \varrho_{p q}\left(\mathbf{u}^{(0)}\right) \\
+\left(b_{k l}-L_{c}^{2} \vartheta_{k l}\right)\left(T^{(0)}-T_{0}\right) \\
\int_{Y} b_{i j} e_{y i j}\left(\mathbf{u}^{(1)}\right) d y=\alpha \delta_{k l}\left(L_{c}^{2} C_{k l p q}^{\varrho}-a_{k l i j} E_{i j}^{p q}\right) \varrho_{p q}\left(\mathbf{u}^{(0)}\right) \\
+L_{c}^{2} S^{*}\left(T^{(0)}-T_{0}\right)
\end{gathered}
$$


With the relations (117) and (118), we can calculate the first term in the right side of (50) to obtain :

$$
\begin{gathered}
\frac{1}{2} \frac{d}{d t} \int_{Y}\left(a_{i j k l} e_{x k l}\left(\mathbf{u}^{(0)}\right)-b_{i j}\left(T^{(0)}-T_{0}\right)\right) e_{y i j}\left(\mathbf{u}^{(1)}\right) d_{y}= \\
\frac{1}{2} L_{c}^{2} \frac{d C_{k l p q}^{\varrho}}{d d} \frac{d d}{d t} \varrho_{p q}\left(\mathbf{u}^{(0)}\right) \varrho_{m n}\left(\mathbf{u}^{(0)}\right) E_{k l}^{m n} \\
-\frac{1}{2} L_{c}^{2} \frac{d \vartheta_{k l}}{d d} \frac{d d}{d t}\left(T^{(0)}-T_{0}\right) \varrho_{m n}\left(\mathbf{u}^{(0)}\right) E_{k l}^{m n} \\
-\frac{1}{2} L_{c}^{2} \frac{d C_{k l p q}^{\varrho}}{d d} \frac{d d}{d t}\left(T^{(0)}-T_{0}\right) \alpha \delta_{k l} \varrho_{p q}\left(\mathbf{u}^{(0)}\right) \\
-\frac{1}{2} L_{c}^{2} \frac{d S^{*}}{d d} \frac{d d}{d t}\left(T^{(0)}-T_{0}\right)^{2} \\
+\frac{1}{2}\left(L_{c}^{2} C_{k l p q}^{\varrho}-a_{k l i j} E_{i j}^{p q}\right)\left(\varrho_{p q}\left(\dot{\mathbf{u}}^{(0)}\right) \varrho_{m n}\left(\mathbf{u}^{(0)}\right)-\varrho_{p q}\left(\mathbf{u}^{(0)}\right) \varrho_{m n}\left(\dot{\mathbf{u}}^{(0)}\right)\right) E_{k l}^{m n} \\
+\frac{1}{2}\left(b_{k l}-L_{c}^{2} \vartheta_{k l}\right)\left(\left(T^{(0)}-T_{0}\right) \varrho_{m n}\left(\dot{\mathbf{u}}^{(0)}\right)+\varrho_{m n}\left(\mathbf{u}^{(0)}\right) \dot{T}^{(0)}\right) E_{k l}^{m n} \\
-\frac{1}{2} \alpha \delta_{k l}\left(L_{c}^{2} C_{k l p q}^{\varrho}-a_{k l i j} E_{i j}^{p q}\right)\left(\left(T^{(0)}-T_{0}\right) \varrho_{p q}\left(\dot{\mathbf{u}}^{(0)}\right)+\varrho_{p q}\left(\mathbf{u}^{(0)}\right) \dot{T}^{(0)}\right) \\
-L_{c}^{2} S^{*} \dot{T}^{(0)}\left(T^{(0)}-T_{0}\right)
\end{gathered}
$$

In the same way, we obtain for the second term in the right side of (50) :

$$
\begin{gathered}
\int_{Y}\left(a_{i j k l} e_{x k l}\left(\dot{\mathbf{u}}^{(0)}\right)-b_{i j} \dot{T}^{(0)}\right) e_{y i j}\left(\mathbf{u}^{(1)}\right) d_{y}= \\
\left(L_{c}^{2} C_{k l p q}^{\varrho}-a_{k l i j} E_{i j}^{p q}\right) \varrho_{p q}\left(\mathbf{u}^{(0)}\right) \varrho_{m n}\left(\dot{\mathbf{u}}^{(0)}\right) E_{k l}^{m n} \\
+\left(b_{k l}-L_{c}^{2} \vartheta_{k l}\right)\left(T^{(0)}-T_{0}\right) \varrho_{m n}\left(\dot{\mathbf{u}}^{(0)}\right) E_{k l}^{m n} \\
-\alpha \delta_{k l}\left(L_{c}^{2} C_{k l p q}^{\varrho}-a_{k l i j} E_{i j}^{p q}\right) \varrho_{p q}\left(\mathbf{u}^{(0)}\right) \dot{T}^{(0)}-L_{c}^{2} S^{*} \dot{T}^{(0)}\left(T^{(0)}-T_{0}\right)
\end{gathered}
$$

Replacing (119-120) in (50) we obtain :

$$
\begin{aligned}
& \frac{1}{L_{c}^{2}} \int_{C Y} \sigma_{i j}^{(0)} N_{j}\left[\dot{u}_{i}^{(1)}\right] d s_{y}-\frac{1}{2 L_{c}^{2}} \frac{d}{d t} \int_{C Y} \sigma_{i j}^{(0)} N_{j}\left[u_{i}^{(1)}\right] d s_{y}-\frac{1}{L_{c}} \frac{d d}{d t} \mathcal{G}^{(1)}= \\
& \frac{d d}{d t}\left(\frac{1}{2} \frac{d C_{k l p q}^{\varrho}}{d d} \varrho_{p q}\left(\mathbf{u}^{(0)}\right) \varrho_{m n}\left(\mathbf{u}^{(0)}\right) E_{k l}^{m n}-\frac{1}{2} \frac{d \vartheta_{k l}}{d d}\left(T^{(0)}-T_{0}\right) \varrho_{m n}\left(\mathbf{u}^{(0)}\right) E_{k l}^{m n}\right. \\
& \left.\quad-\frac{1}{2} \frac{d C_{k l p q}^{\varrho}}{d d}\left(T^{(0)}-T_{0}\right) \alpha \delta_{k l} \varrho_{p q}\left(\mathbf{u}^{(0)}\right)-\frac{1}{2} \frac{d S^{*}}{d d}\left(T^{(0)}-T_{0}\right)^{2}\right) \\
& +\frac{1}{2 L_{c}^{2}} \int_{C Y} a_{i j k l} E_{k l}^{m n} N_{j}\left[\xi_{i}^{p q}\right] d s_{y}\left(\varrho_{p q}\left(\dot{\mathbf{u}}^{(0)}\right) \varrho_{m n}\left(\mathbf{u}^{(0)}\right)-\varrho_{p q}\left(\mathbf{u}^{(0)}\right) \varrho_{m n}\left(\dot{\mathbf{u}}^{(0)}\right)\right) \\
& +\frac{1}{2 L_{c}^{2}} \int_{C Y} a_{i j k l} E_{k l}^{m n} N_{j}\left[\phi_{i}\right] d s_{y}\left(\varrho_{m n}\left(\mathbf{u}^{(0)}\right) \dot{T}^{(0)}-\left(T^{(0)}-T_{0}\right) \varrho_{m n}\left(\dot{\mathbf{u}}^{(0)}\right)\right) \\
& \quad+\frac{1}{2 L_{c}^{2}} \int_{C Y} b_{i j} N_{j}\left[\xi_{i}^{p q}\right] d s_{y}\left(\varrho_{p q}\left(\mathbf{u}^{(0)}\right) \dot{T}^{(0)}-\left(T^{(0)}-T_{0}\right) \varrho_{p q}\left(\dot{\mathbf{u}}^{(0)}\right)\right)
\end{aligned}
$$


With the expressions of $\mathbf{u}^{(1)}$ and $\sigma_{i j}^{(0)}$ we calculate the second term in the left side of $(121)$ as :

$$
\begin{gathered}
\frac{1}{2 L_{c}^{2}} \frac{d}{d t} \int_{C Y} \sigma_{i j}^{(0)} N_{j}\left[u_{i}^{(1)}\right] d s_{y}=\left(\frac{1}{2} \frac{d \mathcal{Z}_{m n p q}^{\varrho 1}}{d d} \varrho_{m n}\left(\mathbf{u}^{(0)}\right) \varrho_{p q}\left(\mathbf{u}^{(0)}\right)\right. \\
\left.+\frac{1}{2}\left(\frac{d \mathcal{Z}_{m n}^{\varrho 2}}{d d}+\frac{d \mathcal{Z}_{m n}^{\varrho 3}}{d d}\right) \varrho_{m n}\left(\mathbf{u}^{(0)}\right)\left(T^{(0)}-T_{0}\right)+\frac{1}{2} \frac{d \mathcal{Z}^{4}}{d d}\left(T^{(0)}-T_{0}\right)^{2}\right) \frac{d d}{d t} \\
+\frac{1}{2} \mathcal{Z}_{m n p q}^{\varrho 1}\left(\varrho_{m n}\left(\dot{\mathbf{u}}^{(0)} \varrho_{p q}\left(\mathbf{u}^{(0)}\right)+\varrho_{m n}\left(\mathbf{u}^{(0)}\right) \varrho_{p q}\left(\dot{\mathbf{u}}^{(0)}\right)\right)\right. \\
+\frac{1}{2}\left(\mathcal{Z}_{m n}^{\varrho 2}+\mathcal{Z}_{m n}^{\varrho 3}\right)\left(\varrho_{m n}\left(\dot{\mathbf{u}}^{(0)}\right)\left(T^{(0)}-T_{0}\right)+\varrho_{m n}\left(\mathbf{u}^{(0)}\right) \dot{T}^{(0)}\right) \\
+\mathcal{Z}^{4} \dot{T}^{(0)}\left(T^{(0)}-T_{0}\right)
\end{gathered}
$$

where

$$
\begin{aligned}
\mathcal{Z}_{m n p q}^{\varrho 1} & =\frac{1}{L_{c}^{2}} \int_{C Y} a_{i j k l}\left(E_{k l}^{m n}+e_{y k l}\left(\boldsymbol{\xi}^{m n}\right)\right) N_{j}\left[\xi_{i}^{p q}\right] d s_{y} \\
\mathcal{Z}_{m n}^{\varrho 2} & =\frac{1}{L_{c}^{2}} \int_{C Y} a_{i j k l}\left(E_{k l}^{m n}+e_{y k l}\left(\boldsymbol{\xi}^{m n}\right)\right) N_{j}\left[\phi_{i}\right] d s_{y} \\
\mathcal{Z}_{m n}^{\varrho 3} & =-\frac{1}{L_{c}^{2}} \int_{C Y}\left(b_{i j}-a_{i j k l} e_{y k l}(\phi)\right) N_{j}\left[\xi_{i}^{m n}\right] d s_{y} \\
\mathcal{Z}^{4} & =-\frac{1}{L_{c}^{2}} \int_{C Y}\left(b_{i j}-a_{i j k l} e_{y k l}(\phi)\right) N_{j}\left[\phi_{i}\right] d s_{y}
\end{aligned}
$$

When the time derivative of $\mathbf{u}^{(1)}$ :

$$
\begin{aligned}
\dot{\mathbf{u}}^{(1)}= & \dot{\boldsymbol{\xi}}^{p q}(\mathbf{y}) \varrho_{p q}\left(\mathbf{u}^{(0)}\right)(\mathbf{x}, t)+\boldsymbol{\xi}^{p q}(\mathbf{y}) \varrho_{p q}\left(\dot{\mathbf{u}}^{(0)}\right)(\mathbf{x}, t) \\
& +\dot{\boldsymbol{\phi}}(\mathbf{y})\left(T^{(0)}(\mathbf{x}, t)-T_{0}\right)+\boldsymbol{\phi}(\mathbf{y}) \dot{T}^{(0)}(\mathbf{x}, t)
\end{aligned}
$$

is used in the first integral in the left member of (121) one obtains :

$$
\begin{aligned}
& \frac{1}{L_{c}^{2}} \int_{C Y} \sigma_{i j}^{(0)} N_{j}\left[\dot{u}_{i}^{(1)}\right] d s_{y}=\left(I_{m n p q}^{\varrho 2} \varrho_{m n}\left(\mathbf{u}^{(0)}\right) \varrho_{p q}\left(\mathbf{u}^{(0)}\right)\right. \\
& \left.\quad+J_{m n}^{\varrho 2} \varrho_{m n}\left(\mathbf{u}^{(0}\right)\left(T^{(0)}-T_{0}\right)-P^{2}\left(T^{(0)}-T_{0}\right)^{2}\right) \frac{d d}{d t} \\
& \quad+\mathcal{Z}_{m n p q}^{\varrho 1} \varrho_{m n}\left(\mathbf{u}^{(0)}\right) \varrho_{p q}\left(\dot{\mathbf{u}}^{(0}\right)+\mathcal{Z}_{m n}^{\varrho 2} \varrho_{m n}\left(\mathbf{u}^{(0}\right) \dot{T}^{(0)} \\
& \quad+\mathcal{Z}_{m n}^{\varrho 3} \varrho_{m n}\left(\dot{\mathbf{u}}^{(0)}\right)\left(T^{(0)}-T_{0}\right)+\mathcal{Z}^{4} \dot{T}^{(0)}\left(T^{(0)}-T_{0}\right)
\end{aligned}
$$

with

$$
\begin{aligned}
I_{m n p q}^{\varrho 2} & =\frac{1}{L_{c}^{2}} \int_{C Y} a_{i j k l}\left(E_{k l}^{m n}+e_{y k l}\left(\boldsymbol{\xi}^{m n}\right)\right) N_{j}\left[\frac{d \xi_{i}^{p q}}{d d}\right] d s_{y} \\
J_{m n}^{\varrho 2} & =\frac{1}{L_{c}^{2}} \int_{C Y} a_{i j k l}\left(E_{k l}^{m n}+e_{y k l}\left(\boldsymbol{\xi}^{m n}\right)\right) N_{j}\left[\frac{d \phi_{i}}{d d}\right] d s_{y} \\
& -\frac{1}{L_{c}^{2}} \int_{C Y}\left(b_{i j}-a_{i j k l} e_{y k l}(\phi)\right) N_{j}\left[\frac{d \xi_{i}^{m n}}{d d}\right] d s_{y} \\
P^{2} & =\frac{1}{2 L_{c}^{2}} \frac{d}{d d} \int_{C Y}\left(b_{i j}-a_{i j k l} e_{y k l}(\phi)\right) N_{j}\left[\frac{d \phi_{i}}{d d}\right] d s_{y}
\end{aligned}
$$


Using the cell problems $(76),(81)$ one can prove that the integral $\int_{C Y} a_{i j k l} e_{y k l}\left(\boldsymbol{\xi}^{m n}\right) N_{j}\left[\xi_{i}^{p q}\right] d s_{y}$ is symmetrical in respect to the pairs $m n$ and $p q$ and we have

$$
\int_{C Y} a_{i j k l} e_{y k l}\left(\xi^{m n}\right) N_{j}\left[\phi_{i}\right] d s_{y}=\int_{C Y} a_{i j k l} e_{y k l}(\phi) N_{j}\left[\xi_{i}^{p q}\right] d s_{y}
$$

Combination with (122) and (128) and substitution in (121) and allow us to obtain the damage relation :

$$
\begin{gathered}
\frac{d d}{d t}\left(\frac{\mathcal{G}^{(1)}}{L_{c}}+\frac{1}{2} \frac{d C_{k l p q}^{\varrho}}{d d} \varrho_{p q}\left(\mathbf{u}^{(0)}\right) \varrho_{m n}\left(\mathbf{u}^{(0)}\right) E_{k l}^{m n}\right. \\
-\frac{1}{2} \frac{d \vartheta_{k l}}{d d}\left(T^{(0)}-T_{0}\right) \varrho_{m n}\left(\mathbf{u}^{(0)}\right) E_{k l}^{m n} \\
-\frac{1}{2} \frac{d C_{k l p q}^{\varrho}}{d d}\left(T^{(0)}-T_{0}\right) \alpha \delta_{k l} \varrho_{p q}\left(\mathbf{u}^{(0)}\right)-\frac{1}{2} \frac{d S^{*}}{d d}\left(T^{(0)}-T_{0}\right)^{2} \\
+I_{m n p q}^{\varrho} \varrho_{m n}\left(\mathbf{u}^{(0)} \varrho_{p q}\left(\mathbf{u}^{(0)}\right)\right. \\
\left.+J_{m n}^{\varrho} \varrho_{m n}\left(\mathbf{u}^{(0)}\right)\left(T^{(0)}-T_{0}\right)-P^{\varrho}\left(T^{(0)}-T_{0}\right)^{2}\right)=0
\end{gathered}
$$

with the effective coefficients

$$
\begin{aligned}
I_{m n p q}^{\varrho} & =\frac{1}{2 L_{c}^{2}} \frac{d}{d d} \int_{C Y} a_{i j k l}\left(E_{k l}^{m n}+e_{y k l}\left(\boldsymbol{\xi}^{m n}\right)\right) N_{j}\left[\xi_{i}^{p q}\right] d s_{y} \\
- & \frac{1}{L_{c}^{2}} \int_{C Y} a_{i j k l}\left(E_{k l}^{m n}+e_{y k l}\left(\boldsymbol{\xi}^{m n}\right)\right) N_{j}\left[\frac{d \xi_{i}^{p q}}{d d}\right] d s_{y} \\
J_{m n}^{\varrho}= & \frac{1}{2 L_{c}^{2}} \frac{d}{d d} \int_{C Y} a_{i j k l}\left(E_{k l}^{m n}+e_{y k l}\left(\boldsymbol{\xi}^{m n}\right)\right) N_{j}\left[\phi_{i}\right] d s_{y} \\
- & \frac{1}{2 L_{c}^{2}} \frac{d}{d d} \int_{C Y}\left(b_{i j}-a_{i j k l} e_{y k l}(\phi)\right) N_{j}\left[\xi_{i}^{m n}\right] d s_{y} \\
- & \frac{1}{L_{c}^{2}} \int_{C Y} a_{i j k l}\left(E_{k l}^{m n}+e_{y k l}\left(\boldsymbol{\xi}^{m n}\right)\right) N_{j}\left[\frac{d \phi_{i}}{d d}\right] d s_{y} \\
& +\int_{C Y}\left(b_{i j}-a_{i j k l} e_{y k l}(\phi)\right) N_{j}\left[\frac{d \xi_{i}^{m n}}{d d}\right] d s_{y} \\
P & =\frac{1}{2 L_{c}^{2}} \frac{d}{d d} \int_{C Y}\left(b_{i j}-a_{i j k l} e_{y k l}(\phi)\right) N_{j}\left[\phi_{i}\right] d s_{y} \\
& -\frac{1}{L_{c}^{2}} \int_{C Y}\left(b_{i j}-a_{i j k l} e_{y k l}(\phi)\right) N_{j}\left[\frac{d \phi_{i}}{d d}\right] d s_{y}
\end{aligned}
$$

In the equation (53) the coefficients $I_{m n p q}$ and $J_{m n}$ are linear combinations of $I_{m n p q}^{\varrho}$ and, respectively, $J_{m n}^{\varrho}$. For $I_{m n p q}$ we get :

$$
\begin{aligned}
I_{1212} & =\frac{\gamma^{2}}{4}\left(I_{2222}^{\varrho}-2 I_{1222}^{\varrho}-2 I_{2212}^{\varrho}+4 I_{1212}^{\varrho}\right) \\
I_{2222} & =4 \gamma^{2}\left(I_{2222}^{\varrho}-I_{1222}^{\varrho}-I_{2212}^{\varrho}+I_{1212}^{\varrho}\right) \\
I_{1222}+I_{2212} & =\gamma^{2}\left(-2 I_{2222}^{\varrho}+3 I_{1222}^{\varrho}+3 I_{2212}^{\varrho}-4 I_{1212}^{\varrho}\right)
\end{aligned}
$$

while the others components $I_{1111}, I_{1112}, I_{1122}, I_{2211}, I_{1211}$ are vanishing. 
Similar combinations are obtained for $I_{m n p q}^{2}$ of equation (61).

$$
\begin{aligned}
I_{1212}^{2} & =\frac{\gamma^{2}}{4}\left(I_{2222}^{\varrho 2}-2 I_{1222}^{\varrho 2}-2 I_{2212}^{\varrho 2}+4 I_{1212}^{\varrho 2}\right) \\
I_{2222}^{2} & =4 \gamma^{2}\left(I_{2222}^{\varrho 2}-I_{1222}^{\varrho 2}-I_{2212}^{\varrho 2}+I_{1212}^{\varrho 2}\right) \\
I_{1222}^{2}+I_{2212}^{2} & =\gamma^{2}\left(-2 I_{2222}^{\varrho 2}+3 I_{1222}^{\varrho 2}+3 I_{2212}^{\varrho 2}-4 I_{1212}^{\varrho 2}\right) \\
I_{1111}^{2} & =I_{1112}^{2}=I_{1122}^{2}=I_{2211}^{2}=I_{1211}^{2}=0
\end{aligned}
$$

Expressions of $J_{m n}$ and $J_{m n}^{2}$ in the definition (53) of $\mathcal{Y}_{f}$ and, respectively, (61) are :

$$
\begin{array}{lll}
J_{11}=-\gamma J_{11}^{\varrho} ; & J_{12}=\frac{\gamma}{2}\left(J_{22}^{\varrho}-2 J_{12}^{\varrho}\right) ; & J_{22}=2 \gamma\left(J_{12}^{\varrho}-J_{22}^{\varrho}\right) \\
J_{11}^{2}=-\gamma J_{11}^{\varrho 2} ; & J_{12}^{2}=\frac{\gamma}{2}\left(J_{22}^{\varrho 2}-2 J_{12}^{\varrho 2}\right) ; & J_{22}^{2}=2 \gamma\left(J_{12}^{\varrho 2}-J_{22}^{\varrho 2}\right)
\end{array}
$$

Finally, the coefficients $\mathcal{Z}_{m n p q}^{1}, \mathcal{Z}_{m n}^{2}$ and $\mathcal{Z}_{m n}^{3}$ in (61) are linear combinations of $\mathcal{Z}_{m n p q}^{\varrho 1}, \mathcal{Z}_{m n}^{\varrho 2}$ and $\mathcal{Z}_{m n}^{\varrho 3}$ correspondingly :

$$
\begin{gathered}
\mathcal{Z}_{1212}^{1}=\frac{\gamma^{2}}{4}\left(\mathcal{Z}_{2222}^{\varrho 1}-2 \mathcal{Z}_{1222}^{\varrho 1}-2 \mathcal{Z}_{2212}^{\varrho 1}+4 \mathcal{Z}_{1212}^{\varrho 1}\right) \\
\mathcal{Z}_{2222}^{1}=4 \gamma^{2}\left(\mathcal{Z}_{2222}^{\varrho 1}-\mathcal{Z}_{1222}^{\varrho 1}-\mathcal{Z}_{2212}^{\varrho 1}+\mathcal{Z}_{1212}^{\varrho 1}\right) \\
\mathcal{Z}_{2212}^{1}=\gamma^{2}\left(-\mathcal{Z}_{2222}^{\varrho 1}+\mathcal{Z}_{1222}^{\varrho 1}+2 \mathcal{Z}_{2212}^{\varrho 1}-2 \mathcal{Z}_{1212}^{\varrho 1}\right) \\
\mathcal{Z}_{1222}^{1}=\gamma^{2}\left(-\mathcal{Z}_{2222}^{\varrho 1}+2 \mathcal{Z}_{1222}^{\varrho 1}+\mathcal{Z}_{2212}^{\varrho 1}-2 \mathcal{Z}_{1212}^{\varrho 1}\right) \\
\mathcal{Z}_{1111}^{1}=\mathcal{Z}_{1112}^{1}=\mathcal{Z}_{1122}^{1}=\mathcal{Z}_{2211}^{1}=\mathcal{Z}_{1211}^{1}=0 . \\
\mathcal{Z}_{11}^{2}=-\gamma \mathcal{Z}_{11}^{\varrho 2} ; \quad \mathcal{Z}_{12}^{2}=\frac{\gamma}{2}\left(\mathcal{Z}_{22}^{\varrho 2}-2 \mathcal{Z}_{12}^{\varrho 2}\right) ; \quad \mathcal{Z}_{22}^{2}=2 \gamma\left(\mathcal{Z}_{12}^{\varrho 2}-\mathcal{Z}_{22}^{\varrho 2}\right) \\
\mathcal{Z}_{11}^{3}=-\gamma \mathcal{Z}_{11}^{\varrho 3} ; \quad \mathcal{Z}_{12}^{3}=\frac{\gamma}{2}\left(\mathcal{Z}_{22}^{\varrho 3}-2 \mathcal{Z}_{12}^{\varrho 3}\right) ; \quad \mathcal{Z}_{22}^{3}=2 \gamma\left(\mathcal{Z}_{12}^{\varrho 3}-\mathcal{Z}_{22}^{\varrho 3}\right)
\end{gathered}
$$

Acknowledgements The research leading to these results has received funding from the European Union's Horizon 2020 Programme (Excellent Science, Marie-Sklodowska-Curie Actions) under REA grant agreement 675602 (Project OUTCOME).

\section{Références}

1. ABAQUS 6.13, Analysis User's Manual. Dassault Systems Simulia Corporation, RI, USA (2013)

2. Andrieux S., Bamberger Y., Marigo J-J., Un modele de materiau microfissure pour les betons et les roches. J. Méc. Theor. Appl., vol. 5, p.471-513 (1986).

3. Archer, J.S., Lesser, A.J., Shear band formation and mode II fracture of polymeric glasses. J. Polym. Sci. B : Polymer Physics 49, 103-114 (2010).

4. Ashby, M.F., Hallam, S.D., The failure of brittle solids containing small cracks under compressive stress states. Acta Metall. 34, 497-510 (1986). 
5. Atiezo, M. K., Gbetchi, K., Dascalu, C., Dynamic shear damage with frictional sliding on microcracks. Engng. Fract. Mech. 236 (2020)

6. Auriault, J.-L., Wave propagation and transient heat transfer in thermoelastic composites. Int. J. Heat Mass Transfer 55, 5972-5978 (2012)

7. Bui, H.D., Ehrlacher, A., Nguyen, Q.S., Propagation de fissure en thermoélasticité dynamique, Journal de Mécanique, 19, 697-723 (1980)

8. Bhatt, H., Rosakis, A., Sammis, G., 2011. A micro-mechanics based constitutive model for brittle failure at high strain rates. J. Appl.Mech. 79(3), 1016-28.

9. Bjerke, T. W., Thermomechanical Behavior of Amorphous Polymers During High-Speed Crack Propagation, Ph. D. Dissertation, University of Delaware, Newark, DE. (2002)

10. Bjerke, T. W., Lambros, J., Theoretical development and experimental validation of a thermally dissipative cohesive zone model for dynamic fracture of amorphous polymers, J. Mech. Phys. Solids 51, 1947-1970 (2003)

11. Bougaut O., Rittel D., On crack-tip cooling during dynamic crack initiation, Int. J. Solids Structures 38, 2517-2532 (2001)

12. Bjerke, T. W., Lambros, J., Heating during shearing and opening dominated dynamic fracture of polymers, Exp. Mech. 42 (1), 107-114 (2002)

13. Camacho G.T., Ortiz M., Computational modelling of impact damage in brittle materials, Int. J. Solids Struct. 33, 2899-2938 (1996)

14. Dascalu, C., Multiscale modeling of rapid failure in brittle solids : branching instabilities. Mech. Mater., 116, 77-89 (2018).

15. Dascalu, C., Bilbie, G., Agiasofitou, E., Damage and size effect in elastic solids : a homogenization approach. Int. J. Solids Struct. 45, 409-430 (2008)

16. Dascalu, C., Gbetchi, K., Dynamic evolution of damage by microcracking with heat dissipation, Int. J. Solids Struct. 174-175, 128 - 144 (2019)

17. Dienes, J. K. , Zuo, Q. H., Kershner, J. D. Impact initiation of explosives and propellants via statistical crack mechanics, J. Mech. Phys. Solids 54(6), 1237-1275 (2006).

18. Duarte, C. A., Grilli, N., Koslowski, M., Effect of initial damage variability on hot-spot nucleation in energetic materials, J. Appl. Phys. 124(2), 025104 (2018).

19. Ene, H.I., On linear thermoelasticity of composite materials, Int. J. Engng. Sci. 21, 443-448 (1983)

20. Fialko, Y., Temperature fields generated by the elastodynamic propagation of shear cracks in the Earth, J. Geophys. Res., 109, B01303 (2004)

21. Fish, J., Practical Multiscaling. John Wiley \& Sons (2013)

22. Francfort, G.A., Homogenization and linear thermoelasticity, SIAM J. Math. Anal. 14, 696-708 (1983)

23. Freund, L.B., Dynamic Fracture Mechanics. Cambridge University Press (1990)

24. Gambarotta, L., Lagomarsino S., A microcrack damage model for brittle materials. Int. J. Solids Struct, 30(2), 177-198 (1993).

25. Grilli, N., Duarte, C. A., Koslowski, M., Dynamic fracture and hot-spot modeling in energetic composites, J. Appl. Phys. 123(6), 065101 (2018).

26. Halm D., Dragon A., An anisotropic model of damage and frictional sliding for brittle materials, European Journal of Mechanics A Solids, Volume : 17, 439-460 (1998).

27. Horii, H., Nemat-Nasser, S., Brittle failure in compression : splitting, faulting and brittleductile transition. Philos. Trans. Roy. Soc. London 319, 337-374 (1986).

28. Huang, C., Subhash, G., Vitton, S.J., A dynamic damage growth model for uniaxial compressive response of rock aggregates. Mech. Mater. 34, 267-277 (2002).

29. Kachanov, L.M., A microcrack model of rock inelasticity - Part I : frictional sliding on microcracks; Part II : propagation of microcracks. Mech. Mater. 1, 19-41 (1982).

30. Keita, O., Dascalu, C., François B., A two-scale model for dynamic damage evolution. J. Mech. Phys. Solids. 64, 170-183 (2014)

31. Lachenbruch AH., Frictional heating, fluid pressure, and the resistance to fault motion. J. Geophys. Res. 85, 6097-6122 1(980). 
32. Lawn, B.R., Marshall D.B., Nonlinear stress-strain curves for solids containing closed cracks with friction. J. Mech. Phys. Vol.46, No. 1, 85-113 (1998).

33. Lee, X., Ju, J.W., Micromechanical Damage Model for Brittle solids - II : Compressive Loadings, J. Engrg. Mech., ASCE, 117, 1515-1536 (1991).

34. Leguillon D., Sanchez-Palencia E., On the behaviour of a cracked elastic body with (or without) friction. J. Mech. Theor. Appl. 1, 195-209 (1982).

35. Nemat-Nasser, S., and Obata M., A microcrack model of dilatancy in brittle material. J. of Applied Mechanics, 55, 24-35 (1988).

36. Nemat-Nasser, S., Deng, H., Strain-rate effect on brittle failure in compression. Acta Metall. Mater. 42, 1013-1024 (1994).

37. Paliwal, B., Ramesh, K. T., An Interacting Micro-Crack Damage Model for Failure of Brittle Materials Under Compression, J. Mech. Phys. Solids, 56(3), 896-923 (2008).

38. Parnell, W.J., 2006. Coupled thermoelasticity in a composite half-space, J. Eng. Math. 56, 1-21 (2006).

39. Ravi-Chandar, K., Lu J., Yang B., Zhu Z., Failure mode transitions in polymers under high strain rate loading. Int. J. Fracture, 101, 33-72 (2000).

40. Ravichandran, G., Subhash, G., A micromechanical model for high strain rate behavior of ceramics. Int. J. Solids Struct. 32, 2627-2646 (1995).

41. Rice JR., Heating, weakening and shear localization in earthquake rupture. Phil. Trans. R. Soc. A 375 : 20160015 (2017).

42. Rittel, D., Experimental investigation of transient thermoelastic effects in dynamic fracture, Int. J. Solids Struct. 35, 2959-2973 (1998)

43. Rittel D., Thermomechanical aspects of dynamic crack initiation. Int. J. Fracture. 99, 199-209 (1999)

44. Rosakis, A. J., O. Samudrala, and D. Coker, Cracks Faster Than the Shear Wave Speed. Science, 284, pp. 1337-1340 (1999).

45. Rosakis, A. J., O. Samudrala, and D. Coker, Intersonic shear crack growth along weak planes. Mat. Res. Innovat., 3, pp. 236-243 (2000).

46. Sanchez-Palencia, E., 1980. Non-homogeneous Media and Vibration Theory. Lecture Notes in Physics, vol.127, Springer, Berlin.

47. Schlüter, A., Kuhn, C., Müller, R., Gross, D., An investigation of intersonic fracture using a phase field model. Arch. Appl. Mech. vol. 86, 321-333 (2016).

48. Slepyan, L.I., Models and Phenomena in Fracture Mechanics, Springer, Berlin. (2002)

49. Sun, N.S., Hsu, T.R., Thermomechanical coupling effects on fractured solids. Int. J. Fract. 78, 67-87 (1996)

50. Shao Y., Zhang Y., Xu X., Zhou Z., Li W., Liu B., Effect of crack pattern on the residual strength of ceramics after quenching, J. Am. Ceram. Soc., 94, 2804-2807 (2011)

51. Telega, J.J., Homogenization of fissured elastic solids in the presence of unilateral conditions and friction. Comput. Mech., 6, 109-127 (1990).

52. Truesdell, C.A., Toupin, R.A., The Classical Field Theories, Handbuch der Physik. Springer-Verlag, Berlin, Germany, Vols. III-1 (1960)

53. Wrzesniak A., Dascalu C., Besuelle P., A two-scale time-dependent model of damage : Influence of micro-cracks friction. European Journal of Mechanics A/Solids vol. 49, 345-361 (2014).

54. Zhu, Q.Z., Shao, J.F., A refined micromechanical damage-friction model with strength prediction for rock-like materials under compression. Int. J. Solids Struct. vol. 60, 75-83 (2015).

55. Zhu, Q.Z., Zhao, L.Y., Shao, J.F., Analytical and numerical analysis of frictional damage in quasi brittle materials. J. Mech. Phys. Solids, vol. 92, 137-163. (2016)

56. Yevtushenko, A.A., Kuciej, M., Influence of the protective strip properties on distribution of the temperature at transient frictional heating, International Journal of Heat and Mass Transfer 52, 376-384 (2009)

57. Yevtushenko, A.A., Kuciej, M., Yevtushenko, O., Three-element model of frictional heating during braking with contact thermal resistance and time-dependent pressure, International Journal of Thermal Sciences 50, 1116 - 1124 (2011) 